\title{
CLOSED IDEALS WITH COUNTABLE HULL IN ALGEBRAS OF ANALYTIC FUNCTIONS SMOOTH UP TO THE BOUNDARY
}

\author{
Cyril Agrafeuil and Mohamed Zarrabi
}

\begin{abstract}
We denote by $\mathbb{T}$ the unit circle and by $\mathbb{D}$ the unit disc. Let $\mathcal{B}$ be a semi-simple unital commutative Banach algebra of functions holomorphic in $\mathbb{D}$ and continuous on $\overline{\mathbb{D}}$, endowed with the pointwise product. We assume that $\mathcal{B}$ is continously imbedded in the disc algebra and satisfies the following conditions:

(H1) The space of polynomials is a dense subset of $\mathcal{B}$.

(H2) $\lim _{n \rightarrow+\infty}\left\|z^{n}\right\|_{\mathcal{B}}^{1 / n}=1$.

(H3) There exist $k \geq 0$ and $C>0$ such that

$$
|1-| \lambda||^{k}\|f\|_{\mathcal{B}} \leq C\|(z-\lambda) f\|_{\mathcal{B}}, \quad(f \in \mathcal{B},|\lambda|<2) .
$$

When $\mathcal{B}$ satisfies in addition the analytic Ditkin condition, we give a complete characterisation of closed ideals $I$ of $\mathcal{B}$ with countable hull $h(I)$, where

$$
h(I)=\{z \in \overline{\mathbb{D}}: f(z)=0, \quad(f \in I)\} .
$$

Then, we apply this result to many algebras for which the structure of all closed ideals is unknown. We consider, in particular, the weighted algebras $\ell^{1}(\omega)$ and $L^{1}\left(\mathbb{R}^{+}, \omega\right)$.
\end{abstract}

\section{Introduction}

We denote by $\mathbb{T}$ the unit circle and by $\mathbb{D}$ the unit disc. Let $n$ be a nonnegative integer. We denote by $a^{n}(\mathbb{D})$ the space of $n$ times continuously differentiable functions on $\overline{\mathbb{D}}$ and holomorphic in $\mathbb{D}$. We set $a^{\infty}(\mathbb{D})=\bigcap_{n \geq 0} a^{n}(\mathbb{D})$. Notice that $a(\mathbb{D})=a^{0}(\mathbb{D})$ is the classical disc algebra.

Let $\mathcal{B}$ be a semi-simple unital commutative Banach algebra of functions holomorphic in $\mathbb{D}$ and continuous on $\overline{\mathbb{D}}$, endowed with the pointwise product. We assume that $\mathcal{B}$ is continuously imbedded in $a(\mathbb{D})$.

2000 Mathematics Subject Classification. 46J20, 46J15.

Key words. Closed ideals, Banach algebras, Ditkin condition. 
We denote by $\alpha: z \mapsto z$ the identity map and we define the following conditions:

(H1) The space of polynomials is a dense subset of $\mathcal{B}$.

(H2) $\lim _{n \rightarrow+\infty}\left\|\alpha^{n}\right\|_{\mathcal{B}}^{1 / n}=1$.

(H3) There exist $k \geq 0$ and $C>0$ such that

$$
|1-| \lambda||^{k}\|f\|_{\mathcal{B}} \leq C\|(\alpha-\lambda) f\|_{\mathcal{B}}, \quad(f \in \mathcal{B},|\lambda|<2) .
$$

Denote by $N_{\mathcal{B}}$ the supremum of integers $n$ such that $\mathcal{B} \subset a^{n}(\mathbb{D})$. As we will see in the next section, condition (H3) implies that $N_{\mathcal{B}}$ is bounded.

We say that $\mathcal{B}$ satisfies the analytic Ditkin condition if for each $z_{0} \in \mathbb{T}$ and each function $f \in \mathcal{B}$ such that $f^{(k)}\left(z_{0}\right)=0,0 \leq k \leq N_{\mathcal{B}}$, there exists a sequence $\left(\tau_{n}\right)_{n \geq 0}$ in $\mathcal{B}$, satisfying the following properties:

(A1) For all $n \geq 0, \tau_{n}\left(z_{0}\right)=0$.

(A2) $\lim _{n \rightarrow+\infty}\left\|\left(1-\tau_{n}\right) f\right\|_{\mathcal{B}}=0$.

And we say that $\mathcal{B}$ satisfies the strong analytic Ditkin condition if, for each $z_{0} \in \mathbb{T}$, the sequence $\left(\tau_{n}\right)_{n>0}$ can be chosen independently of $f$.

Denote by $H^{\infty}$ the space of all holomorphic and bounded functions in $\mathbb{D}$. For $f, g \in H^{\infty}$ we say that $f$ divides $g$ (we write $f \mid g$ ) if $g / f \in H^{\infty}$. Let $I$ be a closed ideal of $\mathcal{B}$. We denote by $U_{I}$ the inner factor of $I$, that is the greatest inner common divisor of all nonzero functions in $I$ (see [14, p. 85]), and we set

$$
h^{k}(I)=\left\{z \in \overline{\mathbb{D}}: f(z)=\cdots=f^{(k)}(z)=0, \quad(f \in I)\right\} \quad\left(0 \leq k \leq N_{\mathcal{B}}\right) .
$$

The ideal $I$ is said to be standard if

(2) $I=\left\{f \in \mathcal{B}: U_{I} \mid f\right.$ and $f^{(k)}=0$ on $\left.h^{k}(I) \cap \mathbb{T}, \quad\left(0 \leq k \leq N_{\mathcal{B}}\right)\right\}$.

In several Banach algebras $\mathcal{B}$ satisfying conditions (H1)-(H3), all closed ideals are standard. This is the case if $\mathcal{B}$ is for example one of the following algebras: $a(\mathbb{D}), a^{n}(\mathbb{D})(n \geq 1), \lambda_{s}(0<s<1)$ and $H_{n}^{p}$ $(n \geq 1,1<p<\infty)$, where

$$
\lambda_{s}=\left\{f \in a(\mathbb{D}):\left|f(z)-f\left(z^{\prime}\right)\right|=o\left(\left(\left|z-z^{\prime}\right|^{s}\right)\right),\left|z-z^{\prime}\right| \rightarrow 0\right\},
$$

and

$$
H_{n}^{p}=\left\{f \text { holomorphic in } \mathbb{D} \text { and } f^{(n)} \in H^{p}\right\},
$$

$H^{p}$ being the classical Hardy space on $\mathbb{D}$ (see respectively $[\mathbf{2 4}]$, [18], [19], $[\mathbf{2 7}])$. For further examples see also $[\mathbf{2 8}]$ and $[\mathbf{3 0}]$. 
But, even in the case where $N_{\mathcal{B}}=0$, we don't know in general the characterisation of all closed ideals of $\mathcal{B}$. For example, in $A^{+}(\mathbb{T})$, the algebra of all functions in $a(\mathbb{D})$ with absolutely convergent Taylor series, it is known only when $h^{0}(I)$ is finite (see [15]), $h^{0}(I)$ is countable (see [3]) or when $h^{0}(I)$ is the Cantor triadic set (see [7]). And in these cases, ideals are standard.

The aim of this paper is to study the structure of closed ideals with countable hull in Banach algebras satisfying conditions (H1)-(H3). The first motivation is Theorem B of [3], which is announced without proof and which concerns ideals with finite hull (see Remark 2.13). The second one is the structure of closed ideals in algebras

$$
A_{s}^{+}(\mathbb{T})=\left\{f \in a(\mathbb{D}):\|f\|_{s}=\sum_{n=0}^{+\infty}|\widehat{f}(n)|(1+n)^{s}<+\infty\right\},
$$

where $s$ is a nonnegative real number. The characterisation of closed ideals with countable hull in $A_{s}^{+}(\mathbb{T})$ is known only in the case $s<1$ (see $[\mathbf{2 3}]$ ), which correspond to the fact that the derivation operator doesn't act on $A_{s}^{+}(\mathbb{T})$.

The main result of this paper (Theorem 2.11) shows that if $\mathcal{B}$ satisfies conditions (H1)-(H3) and the analytic Ditkin condition then every closed ideal $I$ of $\mathcal{B}$ such that $h^{0}(I)$ is at most countable is standard. Notice that a result in the same direction was announced by Faŭvyševskiŭ in [9].

There is no hope to extend this result to all closed ideals of $\mathcal{B}$. Indeed, when $\mathcal{B}=A^{+}(\mathbb{T})$, J. Esterle constructed in $[\mathbf{6}]$ a closed ideal $I$ such that $h^{0}(I)$ is a "thin" set, $U_{I}=1$ and $I \neq\left\{f \in A^{+}(\mathbb{T}): f=0\right.$ on $\left.h^{0}(I)\right\}$.

Then we apply this result (Theorem 2.11) to several concrete Banach algebras. In Section 3, we show that for every $s \geq 0$, all closed ideals in $A_{s}^{+}(\mathbb{T})$, with countable hull, are standard. Then, using a result of H. Hedenmalm (see [13]), we deduce a similar characterisation for a family of closed modular ideals of $L_{s}^{1}\left(\mathbb{R}^{+}\right)$, where

$L_{s}^{1}\left(\mathbb{R}^{+}\right)=\left\{f\right.$ measurable on $\left.\mathbb{R}^{+}:\|f\|_{1, s}=\int_{0}^{+\infty}|f(t)|(1+t)^{s} \mathrm{~d} t<+\infty\right\}$.

In Section 4, we give further examples of Banach algebras where Theorem 2.11 applies.

Acknowledgement. We wish to thank the referee for her/his valuable comments and to have drawn our attention to the work of Faŭvyševskiŭ, where results in the same direction are announced. 


\section{Closed ideals of $\mathcal{B}$}

Throughout this section, $\mathcal{B}$ will denote a semi-simple unital commutative Banach algebra of functions holomorphic in $\mathbb{D}$ and continuous on $\overline{\mathbb{D}}$, endowed with the pointwise product and continuously imbedded in $a(\mathbb{D})$. Without loss of generality we assume that $\|1\|_{\mathcal{B}}=1$. We recall that $N_{\mathcal{B}}$ is the supremum of integers $n$ such that $\mathcal{B} \subset a^{n}(\mathbb{D})$. We will need, in the sequel, some properties of $\mathcal{B}$.

Suppose that $\mathcal{B}$ satisfies conditions (H1) and (H2). We can identify the maximal ideal space of $\mathcal{B}$ with $\overline{\mathbb{D}}$. The identification is given by the map

$$
\overline{\mathbb{D}} \ni z \rightarrow \delta_{z},
$$

where $\delta_{z}$ is the point evaluation at $z: \delta_{z}(f)=f(z), f \in \mathcal{B}$.

Then, for all $z \in \overline{\mathbb{D}}$, we have

$$
|g(z)| \leq\|g\|_{\mathcal{B}}, \quad(g \in \mathcal{B}) .
$$

It follows from the closed graph theorem that the embedding from $\mathcal{B}$ into $a^{N_{\mathcal{B}}}(\mathbb{D})$ is continuous. In particular, for all $z \in \overline{\mathbb{D}}$ and for all $k$, $0 \leq k \leq N_{\mathcal{B}}$, functionals

$$
\chi_{z}^{(k)}: g \mapsto g^{(k)}(z)
$$

are continuous on $\mathcal{B}$.

For $\lambda \in \mathbb{D}$ and $f \in a(\mathbb{D})$, we define the function $R_{\lambda}(f)$ by

$$
R_{\lambda}(f)(z)=\left\{\begin{array}{ll}
\frac{f(z)-f(\lambda)}{z-\lambda} & \text { if } z \in \overline{\mathbb{D}} \backslash\{\lambda\} \\
f^{\prime}(\lambda) & \text { if } z=\lambda
\end{array} .\right.
$$

Clearly $R_{\lambda}$ is a linear map. Notice also that if $\mathcal{B}$ satisfies conditions (H1) and (H2) and if for every $f \in \mathcal{B}, R_{\lambda}(f) \in \mathcal{B}$, then the closed graph theorem asserts that $R_{\lambda}$ is a bounded operator on $\mathcal{B}$.

The following lemma shows that condition (H3) can be equivalently reformulated. We recall that $\alpha: z \mapsto z$ is the identity map.

Lemma 2.1. Suppose that $\mathcal{B}$ satisfies conditions (H1) and (H2). Then, the following conditions are equivalent.

(i) $\mathcal{B}$ satisfies condition (H3).

(ii) There exists $k \geq 0$ such that:

(a) $\left\|\alpha^{n}\right\|_{\mathcal{B}}=\bar{O}\left(n^{k}\right), \quad n \rightarrow+\infty$.

(b) For all $\lambda \in \mathbb{D}, R_{\lambda}$ defines a bounded linear operator on $\mathcal{B}$ and there exists $C>0$ such that

$$
\left\|R_{\lambda}\right\| \leq C(1-|\lambda|)^{-k} \quad(|\lambda|<1) .
$$


(iii) There exists $k \geq 0$ such that:

(c) $\left\|\alpha^{n}\right\|_{\mathcal{B}}=O\left(n^{k}\right), \quad n \rightarrow+\infty$.

(d) $R_{0}$ defines a bounded linear operator on $\mathcal{B}$ and

$$
\left\|R_{0}^{n}\right\|=O\left(n^{k}\right), \quad n \rightarrow+\infty .
$$

Proof: (i) $\Rightarrow$ (ii): Suppose that $\mathcal{B}$ satisfies condition (H3). We begin by showing (b). Let $\lambda \in \mathbb{D}$ and $P$ be a polynomial function. We can write $P-P(\lambda)=(\alpha-\lambda) Q$, where $Q$ is a polynomial function. So $R_{\lambda}(P)=Q$ belongs to $\mathcal{B}$. We deduce from (H3) that

$$
\begin{aligned}
(1-|\lambda|)^{k}\left\|R_{\lambda}(P)\right\|_{\mathcal{B}} & \leq C\|P-P(\lambda)\|_{\mathcal{B}} \\
& \leq 2 C\|P\|_{\mathcal{B}},
\end{aligned}
$$

where $k$ and $C$ are nonnegative constants independent of $P$. Let $\mathcal{P}$ be the space of all polynomials provided with the norm of $\mathcal{B}$. One has just seen that the restriction of $R_{\lambda}$ to $\mathcal{P}$ is a bounded linear operator with norm not exceeding $2 C(1-|\lambda|)^{-k}$. Now, since $\mathcal{B}$ satisfies (H1), $R_{\lambda \mid \mathcal{P}}$ can be extended to a bounded linear operator on $\mathcal{B}$ with norm less than $2 C(1-|\lambda|)^{-k}$. Then, using the fact that functionals $g \mapsto g(z)$, for $z \in \overline{\mathbb{D}}$, are continuous on $\mathcal{B}$, it is easily seen that this extension coincides with $R_{\lambda}$ on $\mathcal{B}$, which finishes the proof of part (b).

Now, let us show that (a) holds. As we have observed before, the set of maximal ideal space can be identified with $\overline{\mathbb{D}}$. Therefore the spectrum of $\alpha$ is equal to $\overline{\mathbb{D}}$. So for all $\lambda$ such that $|\lambda|>1$, the function $\alpha-\lambda$ is invertible in $\mathcal{B}$ and using (1), we get

$$
\left\|(\alpha-\lambda)^{-1}\right\|_{\mathcal{B}} \leq C(|\lambda|-1)^{-k}, \quad(1<|\lambda|<2) .
$$

Let $r>1$, we have $\alpha^{n}=\frac{1}{2 i \pi} \int_{\gamma_{r}} \lambda^{n}(\alpha-\lambda)^{-1} \mathrm{~d} \lambda$, where $\gamma_{r}$ denotes the circle centered in 0 with radius $r$. We deduce from this equality and (3) that

$$
\left\|\alpha^{n}\right\|_{\mathcal{B}} \leq C r^{n+1}(r-1)^{-k} \quad(1<r<2) .
$$

Now, it suffices to take $r=1+1 / n$ to prove $(a)$.

(ii) $\Rightarrow$ (iii): It suffices to show that (d) holds. Let $f \in \mathcal{B}$. For $\lambda \in \mathbb{D}$ we get from part (b) of (ii),

$$
\|f\|_{\mathcal{B}}=\left\|R_{\lambda}((\alpha-\lambda) f)\right\|_{\mathcal{B}} \leq C(1-|\lambda|)^{-k}\|(\alpha-\lambda) f\|_{\mathcal{B}} .
$$


It follows from this that for every $\lambda$ and $\lambda^{\prime}$ in $\mathbb{D}$,

$$
\begin{aligned}
&\left\|R_{\lambda}(f)-R_{\lambda^{\prime}}(f)\right\|_{\mathcal{B}} \\
& \leq C^{2}(1-|\lambda|)^{-k}\left(1-\left|\lambda^{\prime}\right|\right)^{-k}\left\|(\alpha-\lambda)\left(\alpha-\lambda^{\prime}\right)\left(R_{\lambda}(f)-R_{\lambda^{\prime}}(f)\right)\right\|_{\mathcal{B}} \\
& \leq C^{2}(1-|\lambda|)^{-k}\left(1-\left|\lambda^{\prime}\right|\right)^{-k}\left\|\left(\alpha-\lambda^{\prime}\right)(f-f(\lambda))-(\alpha-\lambda)\left(f-f\left(\lambda^{\prime}\right)\right)\right\|_{\mathcal{B}} \\
& \leq C^{2}(1-|\lambda|)^{-k}\left(1-\left|\lambda^{\prime}\right|\right)^{-k} \\
& \times\left(\left|f\left(\lambda^{\prime}\right)-f(\lambda)\right|\|\alpha\|_{\mathcal{B}}+\left|\lambda-\lambda^{\prime}\right|\|f\|_{\mathcal{B}}+\left|\lambda^{\prime} f(\lambda)-\lambda f\left(\lambda^{\prime}\right)\right|\right) .
\end{aligned}
$$

Thus the map $\lambda \rightarrow R_{\lambda}(f)$ is continuous from $\mathbb{D}$ into $\mathcal{B}$. So, for every $n \geq 1$ and $r \in(0,1)$, the integral $\frac{1}{2 i \pi} \int_{\gamma_{r}} \lambda^{-n} R_{\lambda}(f) \mathrm{d} \lambda$ is well defined and belongs to $\mathcal{B}$. We shall now prove that

$$
R_{0}^{n}(f)=\frac{1}{2 i \pi} \int_{\gamma_{r}} \lambda^{-n} R_{\lambda}(f) \mathrm{d} \lambda, \quad(n \geq 1,0<r<1) .
$$

For $\lambda$ and $z$ in $\mathbb{D}$ we have

(5)

$$
\begin{aligned}
R_{\lambda}(f)(z) & =\sum_{m \geq 0} \hat{f}(m) \frac{z^{m}-\lambda^{m}}{z-\lambda} \\
& =\sum_{m \geq 1} \hat{f}(m) \sum_{n=0}^{m-1} \lambda^{n} z^{m-n-1} \\
& =\sum_{n \geq 0}\left(\sum_{m \geq n+1} \hat{f}(m) z^{m-n-1}\right) \lambda^{n} \\
& =\sum_{n \geq 0} R_{0}^{n+1}(f)(z) \lambda^{n} .
\end{aligned}
$$

So the map $\lambda \rightarrow R_{\lambda}(f)(z)$ is expanded in an entire series in $\mathbb{D}$ and its Taylor coefficients are $\left(R_{0}^{n+1}(f)(z)\right)_{n \geq 0}$. Hence

$$
R_{0}^{n}(f)(z)=\frac{1}{2 i \pi} \int_{\gamma_{r}} \lambda^{-n} R_{\lambda}(f)(z) \mathrm{d} \lambda, \quad(|z|<1,0<r<1, n \geq 1) .
$$

Since the linear map $\delta_{z}: f \rightarrow f(z)$ is continuous on $\mathcal{B}$, we get

$$
R_{0}^{n}(f)(z)=\left(\frac{1}{2 i \pi} \int_{\gamma_{r}} \lambda^{-n} R_{\lambda}(f) \mathrm{d} \lambda\right)(z), \quad(|z|<1,0<r<1, n \geq 1),
$$


which proves (4). Therefore

$$
\left\|R_{0}^{n}\right\| \leq \frac{1}{2 \pi} \int_{0}^{2 \pi} r^{-n+1}\left\|R_{r e^{i \theta}}(f)\right\|_{\mathcal{B}} \mathrm{d} \theta \leq C r^{-n+1}(1-r)^{-k} .
$$

For $r=1-\frac{1}{n}$, we see that (d) holds.

(iii) $\Rightarrow$ (i): For $|\lambda|<1$, the series $\sum_{n>0} \lambda^{n} R_{0}^{n+1}(f)$ is absolutely convergent in $\mathcal{B}$. Then, by (5), we deduce that $R_{\lambda}(f)=\sum_{n \geq 0} \lambda^{n} R_{0}^{n+1}(f)$ and $R_{\lambda}(f) \in \mathcal{B}$. Therefore there exist constants $C$ and $C^{\prime}$ such that

$$
\begin{aligned}
\left\|R_{\lambda}(f)\right\|_{\mathcal{B}} & \leq \sum_{n \geq 0}|\lambda|^{n}\left\|R_{0}^{n+1}(f)\right\|_{\mathcal{B}} \\
& \leq C\left(\sum_{n \geq 0}|\lambda|^{n}(1+n)^{k}\right)\|f\|_{\mathcal{B}} \leq C^{\prime}(1-|\lambda|)^{-k-1}\|f\|_{\mathcal{B}} .
\end{aligned}
$$

Using this for $(\alpha-\lambda) f$, we obtain

$$
\|f\|_{\mathcal{B}}=\left\|R_{\lambda}((\alpha-\lambda) f)\right\|_{\mathcal{B}} \leq C^{\prime}(1-|\lambda|)^{-k-1}\|(\alpha-\lambda) f\|_{\mathcal{B}} .
$$

Now, let $|\lambda|>1$. The function $\alpha-\lambda$ is invertible in $\mathcal{B}$ and we have

$$
\|f\|_{\mathcal{B}} \leq\left\|(\alpha-\lambda)^{-1}\right\|_{\mathcal{B}}\|(\alpha-\lambda) f\|_{\mathcal{B}}, \quad(f \in \mathcal{B}) .
$$

Then, it suffices to expand $(\alpha-\lambda)^{-1}$ in series and use (c) to see that there exists a constant $C$ such that

(7) $\left\|(\alpha-\lambda)^{-1}\right\|_{\mathcal{B}} \leq \sum_{n \geq 0}\left\|\alpha^{n}\right\|_{\mathcal{B}}|\lambda|^{-n-1} \leq C(|\lambda|-1)^{-k-1}, \quad(1<|\lambda|<2)$.

It follows from (6) and (7) that

$$
\|f\|_{\mathcal{B}} \leq C(|\lambda|-1)^{-k-1}\|(\alpha-\lambda) f\|_{\mathcal{B}}, \quad(1<|\lambda|<2) .
$$

Thus condition (H3) is satisfied.

Remark 2.2. Suppose that $\mathcal{B}$ satisfies conditions (H1) and (H2). Condition (a) in (ii) of Lemma 2.1 can be equivalently reformulated. Assume that $a^{\infty}(\mathbb{D}) \subset \mathcal{B}$. By the closed graph theorem the imbedding $a^{\infty}(\mathbb{D}) \hookrightarrow \mathcal{B}$ is continuous. So there exist $k \geq 0$ and a constant $C>0$ such that $\|f\|_{\mathcal{B}} \leq C\|f\|_{a^{k}(\mathbb{D})}$, whenever $f \in a^{\infty}(\mathbb{D})$. In particular we have $\left\|\alpha^{n}\right\|_{\mathcal{B}}=O\left(n^{k}\right), n \rightarrow \infty$. This implies that $a^{k+2}(\mathbb{D}) \subset \mathcal{B}$. Now it is easily seen that the following conditions are equivalent:

(a) There exists $k \geq 0$ such that $\left\|\alpha^{n}\right\|_{\mathcal{B}}=O\left(n^{k}\right), n \rightarrow+\infty$.

(a') $a^{\infty}(\mathbb{D}) \subset \mathcal{B}$.

(a") There exists $k \geq 0$ such that $a^{k}(\mathbb{D}) \subset \mathcal{B}$. 
To show the main result of this paper we begin with the characterisation of closed ideals $I$ such that $U_{I}=1$ and $h^{0}(I)$ is reduced to a single point. For this we need some lemmas. We denote by $\mathcal{C}^{k}(\mathbb{T})$ the space of $k$ times continuously differentiable functions on $\mathbb{T}$.

Let $f \in \mathcal{B}$. If $z \in \overline{\mathbb{D}}$, we set

$$
k_{f}(z)= \begin{cases}\sup \left\{k \geq 0: f \in \mathcal{C}^{k}(\mathbb{T}) \text { and } f^{(k)}(z)=0\right\} & \text { if } z \in \mathbb{T} \\ \sup \left\{k \geq 0: f^{(k)}(z)=0\right\} & \text { if } z \in \mathbb{D}\end{cases}
$$

and

$$
\begin{aligned}
m_{f}(z)=\sup \{m \geq 1: f & =f_{1} \ldots f_{m} \\
& \text { with } \left.f_{i} \in \mathcal{B} \text { and } f_{i}(z)=0 \quad(1 \leq i \leq m)\right\},
\end{aligned}
$$

with the understanding that $\sup \emptyset=-\infty$. We remark that $k_{f}(z) \geq$ $\min \left(m_{f}(z)-1, N_{\mathcal{B}}\right)$. Also if $z \in \mathbb{D}$ and $\mathcal{B}$ satisfies conditions (H1)-(H3), then $k_{f}(z)=m_{f}(z)-1$.

Lemma 2.3. Suppose that $\mathcal{B}$ satisfies conditions (H1) and (H2). Let $f \in \mathcal{B}, z_{0} \in \mathbb{T}$ and $k$ an integer with $0 \leq k \leq N_{\mathcal{B}}$. If $k_{f}\left(z_{0}\right) \geq k$, then there exists a sequence $\left(P_{m}\right)_{m \geq 0}$ of polynomial functions such that

$$
\lim _{m \rightarrow+\infty}\left\|f-\left(\alpha-z_{0}\right)^{k+1} P_{m}\right\|_{\mathcal{B}}=0 .
$$

Proof: Since polynomial functions are dense in $\mathcal{B}$, there exists a sequence $\left(Q_{m}\right)_{m \geq 0}$ of polynomial functions such that

$$
\lim _{m \rightarrow+\infty}\left\|f-Q_{m}\right\|_{\mathcal{B}}=0 .
$$

For all $m \geq 0$, set

$$
R_{m}=Q_{m}-Q_{m}\left(z_{0}\right)-Q_{m}^{\prime}\left(z_{0}\right)\left(\alpha-z_{0}\right)-\cdots-\frac{Q_{m}^{(k)}\left(z_{0}\right)}{k !}\left(\alpha-z_{0}\right)^{k} .
$$

For every integer $j, 0 \leq j \leq N_{\mathcal{B}}$, the functional $g \mapsto g^{(j)}\left(z_{0}\right)$ is continuous on $\mathcal{B}$, so $\lim _{m \rightarrow+\infty} Q_{m}^{(j)}\left(z_{0}\right)=f^{(j)}\left(z_{0}\right)=0$. Hence, we have

$$
\lim _{m \rightarrow+\infty}\left\|f-R_{m}\right\|_{\mathcal{B}}=0 .
$$

Now, since for all $m \geq 0$, the polynomial function $R_{m}$ vanishes, with all its derivatives of order less or equal than $k$, at $z_{0}$, there exists a polynomial function $P_{m}$ such that

$$
R_{m}=\left(\alpha-z_{0}\right)^{k+1} P_{m},
$$

which concludes the proof. 
Lemma 2.4. Suppose that $\mathcal{B}$ satisfies conditions (H1) and (H2). Let $f \in \mathcal{B}, z_{0} \in \mathbb{T}$ and $k$ be a nonnegative integer. If $m_{f}\left(z_{0}\right) \geq k$, then there exists a sequence $\left(P_{m}\right)_{m \geq 0}$ of polynomial functions such that

$$
\lim _{m \rightarrow+\infty}\left\|f-\left(\alpha-z_{0}\right)^{k} P_{m}\right\|_{\mathcal{B}}=0 \text {. }
$$

Proof: Assume that $m_{f}\left(z_{0}\right) \geq k$. There exist functions $f_{1}, \ldots, f_{k}$ in $\mathcal{B}$ vanishing at $z_{0}$ such that $f=f_{1} \ldots f_{k}$. It follows from Lemma 2.3 that for each $i \in\{1, \ldots, k\}$ there exists a sequence of polynomials $\left(P_{i, m}\right)_{m}$ such that $\lim _{m \rightarrow+\infty}\left\|f_{i}-\left(\alpha-z_{0}\right) P_{i, m}\right\|_{\mathcal{B}}=0$. If for each $m$, we set $P_{m}=$ $P_{1, m} \ldots P_{k, m}$ then we have clearly $\lim _{m \rightarrow+\infty}\left\|f-\left(\alpha-z_{0}\right)^{k} P_{m}\right\|_{\mathcal{B}}=0$.

Lemma 2.5. Suppose that $\mathcal{B}$ satisfies conditions (H1) and (H2) and the analytic Ditkin condition. Let $z_{0} \in \mathbb{T}$ and $f \in \mathcal{B}$ such that $k_{f}\left(z_{0}\right) \geq N_{\mathcal{B}}$. Then, for all $m \geq 1$, there exists a sequence $\left(\sigma_{n}\right)_{n \geq 0}$ included in $\mathcal{B}$ such that for each $n, m_{\sigma_{n}}\left(z_{0}\right) \geq m$, and

$$
\lim _{n \rightarrow+\infty}\left\|\sigma_{n} f-f\right\|_{\mathcal{B}}=0 \text {. }
$$

Proof: Since $\mathcal{B}$ satisfies the analytic Ditkin condition, there exists a sequence $\left(\tau_{n}\right)_{n \geq 0}$ included in $\mathcal{B}$ satisfying conditions (A1) and (A2) associated with the function $f$. This gives the conclusion in the case where $m=1$. Now, suppose that $m \geq 2$. We construct by induction on $k$ a sequence $\left(\tau_{n_{1}, \ldots, n_{k}}\right)_{\left(n_{1}, \ldots, n_{k}\right) \in \cup_{1 \leq k \leq m} \mathbb{N}^{k}}$ such that for every $k \in\{1, \ldots, m-1\}$ and $\left(n_{1}, \ldots, n_{k}\right) \in \mathbb{N}^{k}$ the sequence $\left(\tau_{n_{1}, \ldots, n_{k}, n}\right)_{n \geq 0}$ satisfies conditions (A1) and (A2) related to the function $\tau_{n_{1}, \ldots, n_{k}} f$, that is

and

$$
\tau_{n_{1}, \ldots, n_{k}, n}\left(z_{0}\right)=0, \quad \text { for every } n \geq 0
$$

$$
\lim _{n \rightarrow+\infty}\left\|\tau_{n_{1}, \ldots, n_{k}, n} \tau_{n_{1}, \ldots, n_{k}} f-\tau_{n_{1}, \ldots, n_{k}} f\right\|_{\mathcal{B}}=0 .
$$

To simplify, we assume that $m=2$ (the general case can be proved exactly in the same way). It is easily seen that there exist $\left(\varphi_{1}(n)\right)_{n \geq 0}$ and $\left(\varphi_{2}(n)\right)_{n \geq 0}$ two increasing sequences of positive integers such that, for all $n \geq 0$,

$$
\begin{gathered}
\left\|\tau_{\varphi_{1}(n)} f-f\right\|_{\mathcal{B}} \leq \frac{1}{n+1} \\
\text { and }\left\|\tau_{\varphi_{1}(n), \varphi_{2}(n)} \tau_{\varphi_{1}(n)} f-\tau_{\varphi_{1}(n)} f\right\|_{\mathcal{B}} \leq \frac{1}{n+1}
\end{gathered}
$$

Now, set

$$
\sigma_{n}=\tau_{\varphi_{1}(n), \varphi_{2}(n)} \tau_{\varphi_{1}(n)} \quad(n \geq 0)
$$


Then, as

$$
\left\|\sigma_{n} f-f\right\|_{\mathcal{B}} \leq\left\|\tau_{\varphi_{1}(n), \varphi_{2}(n)} \tau_{\varphi_{1}(n)} f-\tau_{\varphi_{1}(n)} f\right\|_{\mathcal{B}}+\left\|\tau_{\varphi_{1}(n)} f-f\right\|_{\mathcal{B}},
$$

we have

$$
\lim _{n \rightarrow+\infty}\left\|\sigma_{n} f-f\right\|_{\mathcal{B}}=0 .
$$

And since for all $n \geq 0, \tau_{\varphi_{1}(n), \varphi_{2}(n)}\left(z_{0}\right)=0$ and $\tau_{\varphi_{1}(n)}\left(z_{0}\right)=0$, we have $m_{\sigma_{n}}\left(z_{0}\right) \geq 2$.

Let $f \in a(\mathbb{D})$. We denote by $U_{f}$ its inner factor. We have $U_{f}=B_{f} S_{f}$, where $B_{f}$ is the Blaschke product constructed with zeros of $f$ in $\mathbb{D}$, and $S_{f}$ its singular inner factor, associated with a singular measure $\mu_{f}$.

Let $I$ be a nonzero closed ideal of $\mathcal{B}$ and $z \in \overline{\mathbb{D}}$. We set

$$
k_{I}(z)=\inf \left\{k_{f}(z): f \in I\right\}
$$

and (what have already been defined in the Introduction)

$$
h^{k}(I)=\left\{z \in \overline{\mathbb{D}}: k_{I}(z) \geq k\right\}, \quad\left(0 \leq k \leq N_{\mathcal{B}}\right)
$$

We recall that $U_{I}$ stands for the inner factor of $I$, that is the greatest common divisor of all nonzero functions in $I$ (see $\left[\mathbf{1 4}\right.$, p. 85]). Let $B_{I}$ be the Blaschke product constructed with elements of $h^{0}(I) \cap \mathbb{D}$ (taking multiplicity into account). Then $U_{I}=B_{I} S_{I}$, where $S_{I}$ is the singular inner function associated with $U_{I}$. We denote by $\mu_{I}$ the singular positive measure which defines $S_{I}$.

The following lemma appears in $[7]$ in the particular case where $\mathcal{B}=$ $A^{+}(\mathbb{T})$. Since $\mathcal{B} \subset a(\mathbb{D})$, the proof uses exactly same arguments, so we state it without proof.

Lemma 2.6. Assume that $\mathcal{B}$ satisfies conditions (H1) and (H2) and let $I$ be a nonzero closed ideal of $\mathcal{B}$. Then, there exists a sequence $\left(f_{n}\right)_{n \geq 0}$ of nonzero functions in I such that

$$
\lim _{n \rightarrow+\infty}\left\|\mu_{f_{n}}-\mu_{I}\right\|=0 .
$$

Suppose that $\mathcal{B}$ satisfies conditions $(\mathrm{H} 1)-(\mathrm{H} 3)$. Let $I$ be a closed ideal of $\mathcal{B}$ such that $h^{0}(I) \subset \mathbb{T}$. Denote by $\pi_{I}$ the canonical surjection from $\mathcal{B}$ onto $\mathcal{B} / I$. Let $T$ be the operator on $\mathcal{B} / I$ defined by

$$
T_{I}: \pi_{I}(f) \longmapsto \pi_{I}(\alpha) \pi_{I}(f),
$$

where $\alpha: z \mapsto z$ is the identity map; $T_{I}$ is a bounded linear operator on $\mathcal{B} / I$ such that $\operatorname{Sp}\left(T_{I}\right)=h^{0}(I)$.

Let $f \in \mathcal{B}$ and $\lambda \in \mathbb{D}$. We have $(\alpha-\lambda) R_{\lambda}(f)=f-f(\lambda)$, which implies that

$$
\pi_{I}\left(R_{\lambda}(f)\right)=\left(\pi_{I}(\alpha)-\lambda\right)^{-1}\left(\pi_{I}(f)-f(\lambda)\right)
$$


Suppose that $f \in I$. We have $\pi_{I}\left(R_{\lambda}(f)\right)=-f(\lambda)\left(\pi_{I}(\alpha)-\lambda\right)^{-1}$. Therefore we get

$$
\begin{aligned}
\left\|f(\lambda)\left(\lambda-\pi_{I}(\alpha)\right)^{-1}\right\| & =\left\|\pi_{I}\left(R_{\lambda}(f)\right)\right\| \\
& \leq\left\|R_{\lambda}(f)\right\|_{\mathcal{B}} .
\end{aligned}
$$

By Lemma 2.1, there exist nonnegative contants $C$ and $k$ such that

$$
\left\|f(\lambda)\left(\lambda-T_{I}\right)^{-1}\right\| \leq C(1-|\lambda|)^{-k}\|f\|_{\mathcal{B}} .
$$

Denote by $\nu_{f}\left(\right.$ resp. $\left.\nu_{I}\right)$ the discrete part of the singular measure $\mu_{f}$ (resp. $m_{I}$ ). Then, we deduce from (9) and from Lemma $5 \mathrm{c}$ of $[2]$ that, for all $\varepsilon>0$,

$$
\left\|\left(\lambda-T_{I}\right)^{-1}\right\|=O\left((1-|\lambda|)^{-k} e^{\frac{2\left\|\nu_{f}\right\|+\varepsilon}{1-|\lambda|}}\right), \quad|\lambda| \rightarrow 1^{-} .
$$

This shows that

$$
\limsup _{|\lambda| \rightarrow 1-}(1-|\lambda|) \log ^{+}\left\|\left(\lambda-T_{I}\right)^{-1}\right\| \leq 2\left\|\nu_{f}\right\| .
$$

Notice that $\nu_{f}-\nu_{I}$ is the discrete part of $\mu_{f}-\mu_{I}$. It follows from Lemma 2.6 that there exists $\left(f_{n}\right)_{n}$ in $I$ such that $\lim _{n \rightarrow \infty}\left\|\nu_{f_{n}}-\nu_{I}\right\|=0$. So

$$
\limsup _{|\lambda| \rightarrow 1-}(1-|\lambda|) \log ^{+}\left\|\left(\lambda-T_{I}\right)^{-1}\right\| \leq 2\left\|\nu_{I}\right\| .
$$

Now, the following lemma gives the characterisation of closed ideals $I$ of $\mathcal{B}$ such that $U_{I}=1$ and $h^{0}(I)=\left\{z_{0}\right\}, z_{0} \in \mathbb{T}$.

Lemma 2.7. Suppose that $\mathcal{B}$ satisfies conditions (H1)-(H3) and the analytic Ditkin condition. Let $z_{0} \in \mathbb{T}$ and $I$ be a closed ideal of $\mathcal{B}$ such that $U_{I}=1$ and $h^{0}(I)=\left\{z_{0}\right\}$. Then

$$
I=\left\{f \in \mathcal{B}: f^{(k)}\left(z_{0}\right)=0, \quad\left(0 \leq k \leq k_{I}\left(z_{0}\right)\right)\right\} .
$$

Proof: To simplify notations, we put $k_{I}=k_{I}\left(z_{0}\right)$. It follows from Lemma 2.3 that

$$
I \subset \overline{\left(\alpha-z_{0}\right)^{k_{I}+1} \mathcal{B}},
$$

where the closure is taken for the norm in $\mathcal{B}$. Now, we are interested in showing the other inclusion. By Lemma 2.1, there exists a nonnegative integer $k$ such that $\left\|\alpha^{n}\right\|=O\left(n^{k}\right), n \rightarrow \infty$. The operator $T$ defined on $\mathcal{B} / I$ by

$$
T: \pi_{I}(f) \longmapsto \pi_{I}(\alpha) \pi_{I}(f),
$$


satisfies $\operatorname{Sp} T=\left\{z_{0}\right\}$ and $\left\|T^{n}\right\|=O\left(n^{k}\right), n \rightarrow+\infty$. On the other hand, we deduce from (10) that, for all $\varepsilon>0$,

$$
\left\|(z-T)^{-1}\right\|=O\left(e^{\frac{\varepsilon}{1-|z|}}\right), \quad|z| \rightarrow 1^{-} .
$$

We obtain by Lemma 2 of $[\mathbf{2}]$ that, for all $\varepsilon>0$,

$$
\left\|T^{-n}\right\|=O\left(e^{\varepsilon \sqrt{n}}\right), \quad n \rightarrow+\infty .
$$

Then, it follows from Corollary 1 of $[2]$ that $\left(T-z_{0}\right)^{k+1}=0$, which means that

$$
\left(\alpha-z_{0}\right)^{k+1} \in I .
$$

Denote by $k_{0}$ the smallest of nonnegative integers $p$ such that $(\alpha-$ $\left.z_{0}\right)^{p+1} \in I$. We are going to prove that $k_{0} \leq k_{I}$. Since $\mathcal{B}$ satisfies the analytic Ditkin condition, we deduce from Lemma 2.5 that there exists a sequence $\left(\sigma_{n}\right)_{n \geq 0}$ included in $\mathcal{B}$ such that $m_{\sigma_{n}}\left(z_{0}\right) \geq k+1$ and

$$
\lim _{n \rightarrow+\infty}\left\|\sigma_{n}\left(\alpha-z_{0}\right)^{N_{\mathcal{B}}+1}-\left(\alpha-z_{0}\right)^{N_{\mathcal{B}}+1}\right\|_{\mathcal{B}}=0 .
$$

Since $m_{\sigma_{n}}\left(z_{0}\right) \geq k+1$, we deduce from Lemma 2.4 that there exists a sequence $\left(P_{n, k}\right)_{k \geq 0}$ of functions included in $\mathcal{B}$ such that, for all $n \geq 0$,

$$
\lim _{k \rightarrow+\infty}\left\|\sigma_{n}-\left(\alpha-z_{0}\right)^{k+1} P_{n, k}\right\|_{\mathcal{B}}=0 .
$$

Since $\left(\alpha-z_{0}\right)^{k+1} \in I$, it follows from this equality that, for all $n \geq 0$, $\sigma_{n} \in I$. Then, we deduce from (12) that $\left(\alpha-z_{0}\right)^{N_{\mathcal{B}}+1} \in I$, and so $k_{0} \leq N_{\mathcal{B}}$.

Let $f \in I$ and set

$$
g=f-f\left(z_{0}\right)-\frac{f^{\prime}\left(z_{0}\right)}{1 !}\left(\alpha-z_{0}\right)-\cdots-\frac{f^{\left(k_{0}\right)}\left(z_{0}\right)}{\left(k_{0}\right) !}\left(\alpha-z_{0}\right)^{k_{0}} .
$$

We have $k_{g}\left(z_{0}\right) \geq k_{0}$. Since $\left(\alpha-z_{0}\right)^{k_{0}+1} \in I$ and $k_{0} \leq N_{B}$, Lemma 2.3 implies that $g \in I$. Applying $\pi_{I}$ in (13), we get

(14) $f\left(z_{0}\right)+f^{\prime}\left(z_{0}\right)\left(\pi_{I}(\alpha)-z_{0}\right)+\cdots+\frac{f^{\left(k_{0}\right)}\left(z_{0}\right)}{\left(k_{0}\right) !}\left(\pi_{I}(\alpha)-z_{0}\right)^{k_{0}}=0$.

Now, using the definition of $k_{0}$, one can easily prove that the family $\left(\left(\pi_{I}(\alpha)-z_{0}\right)^{k}\right)_{0 \leq k \leq k_{0}}$ is linearly independent. So we deduce from (14) that for all $k \in\left\{0, \ldots, k_{0}\right\}, f^{(k)}\left(z_{0}\right)=0$. Hence, for all $f \in I, k_{f}\left(z_{0}\right) \geq$ $k_{0}$, and so $k_{I} \geq k_{0}$. Consequently, $\left(\alpha-z_{0}\right)^{k_{I}+1} \in I$, which proves that

$$
\overline{\left(\alpha-z_{0}\right)^{k_{I}+1} \mathcal{B}} \subset I .
$$


Finally, we have proved that

$$
\begin{aligned}
I & =\overline{\left(\alpha-z_{0}\right)^{k_{I}+1} \mathcal{B}} \\
& =\left\{f \in \mathcal{B}: f^{(k)}\left(z_{0}\right)=0 \quad\left(0 \leq k \leq k_{I}\right)\right\}
\end{aligned}
$$

(the second equality follows from Lemma 2.3).

Lemma 2.8. Let $\mu$ be a singular positive measure on $\mathbb{T}$, and $S_{\mu}$ the singular inner function associated with $\mu$. Let $p$ be a nonnegative integer and $f \in a^{p}(\mathbb{D})$ such that $S_{\mu} \mid f$. Then, we have

$$
f^{(k)}=0 \text { on } \operatorname{Supp} \mu, \quad(0 \leq k \leq p),
$$

where $\operatorname{Supp} \mu$ denotes the support of the measure $\mu$.

Proof: By hypothesis, there exists $g \in H^{\infty}(\mathbb{D})$ such that $S_{\mu} g=f$. Now, if $\lambda \in \mathbb{T}$, we deduce from $[\mathbf{2 1}$, p. 63] that

$$
\lambda \in \operatorname{Supp} \mu \Longleftrightarrow \liminf _{\mathbb{D} \ni \xi \rightarrow \lambda} S_{\mu}(\xi)=0 .
$$

Since $g$ is bounded, if $\lambda \in \operatorname{Supp} \mu$, we have

$$
f(\lambda)=\liminf _{\mathbb{D} \ni \xi \rightarrow \lambda} f(\xi)=\liminf _{\mathbb{D} \ni \xi \rightarrow \lambda} S_{\mu}(\xi) g(\xi)=0,
$$

which proves that $f=0$ on $\operatorname{Supp} \mu$. Then, if $\lambda$ is an accumulation point of Supp $\mu$, we have $f^{(k)}(\lambda)=0$ for all $k \in\{0, \ldots, p\}$. Now, suppose that $\lambda$ is an isolated point of $\operatorname{Supp} \mu$. Set $a=\mu(\{\lambda\})$ and $\nu=\mu-a \delta_{\lambda}$, where $\delta_{\lambda}$ denotes the Dirac measure at $\lambda$. We have $a>0$ and we can write $f=e^{a \frac{\alpha+\lambda}{\alpha-\lambda}} h$, where $h=S_{\nu} g \in H^{\infty}(\mathbb{D})$. We have already proved that $f(\lambda)=0$. Suppose that $f(\lambda)=f^{\prime}(\lambda)=\cdots=f^{(k-1)}(\lambda)=0$ for some $k \in\{1, \ldots, p\}$. Then, we have

$$
\begin{aligned}
f^{(k)}(\lambda) & =k ! \lim _{r \rightarrow 1^{-}} \frac{f(r \lambda)}{(r \lambda-\lambda)^{k}} \\
& =k ! \lim _{r \rightarrow 1^{-}} \frac{e^{a \frac{r \lambda+\lambda}{r \lambda-\lambda}}}{(r \lambda-\lambda)^{k}} h(r \lambda) \\
& =0 .
\end{aligned}
$$

Hence, an induction on $k$ proves that $f^{(k)}(\lambda)=0$ for all $k \in\{0, \ldots, p\}$. This concludes the proof. 
Lemma 2.9. Assume that $\mathcal{B}$ satisfies conditions (H1)-(H3). Let $f \in \mathcal{B}$ and let $\psi$ be an infinitely differentiable function on $\mathbb{T}$. Then the Cauchy transform of $\psi f_{\mid \mathbb{T}}$ defined by

$$
\mathcal{C}(\psi f)(z)=\frac{1}{2 i \pi} \int_{\mathbb{T}} \frac{\psi(\zeta) f(\zeta)}{\zeta-z} \mathrm{~d} \zeta, \quad(z \in \mathbb{D}),
$$

belongs to $\mathcal{B}$.

Proof: Let $z \in \mathbb{D}$. Cauchy formulae shows that for $n \geq 0$,

$$
\frac{1}{2 i \pi} \int_{\mathbb{T}} \frac{\zeta^{n} f(\zeta)}{\zeta-z} \mathrm{~d} \zeta=z^{n} f(z)
$$

and that for $n<0$,

$$
\begin{aligned}
\frac{1}{2 i \pi} \int_{\mathbb{T}} \frac{\zeta^{n} f(\zeta)}{\zeta-z} \mathrm{~d} \zeta & =\frac{1}{2 i \pi} \int_{\mathbb{T}} \frac{\zeta^{n}\left(f(\zeta)-\hat{f}(0)-\hat{f}(1) \zeta-\cdots-\hat{f}(-n-1) \zeta^{-n-1}\right)}{\zeta-z} \mathrm{~d} \zeta \\
& =\frac{1}{2 i \pi} \int_{\mathbb{T}} \frac{R_{0}^{-n}(f)(\zeta)}{\zeta-z} \mathrm{~d} \zeta \\
& =R_{0}^{-n}(f)(z) .
\end{aligned}
$$

So

$$
\begin{aligned}
\mathcal{C}(\psi f)(z) & =\frac{1}{2 i \pi} \sum_{n \in \mathbb{Z}} \hat{\psi}(n) \int_{\mathbb{T}} \frac{\zeta^{n} f(\zeta)}{\zeta-z} \mathrm{~d} \zeta \\
& =\sum_{n \geq 0} \hat{\psi}(n) z^{n} f(z)+\sum_{n<0} \hat{\psi}(n) R_{0}^{-n}(f)(z) .
\end{aligned}
$$

Since $\psi$ is infinitely differentiable, it follows from Lemma 2.1, that the series

$$
\sum_{n \geq 0}|\hat{\psi}(n)|\left\|\alpha^{n} f\right\|_{\mathcal{B}}+\sum_{n<0}|\hat{\psi}(n)|\left\|R_{0}^{-n}(f)\right\|_{\mathcal{B}}
$$

converges. So $\sum_{n \geq 0} \hat{\psi}(n) z^{n} f+\sum_{n<0} \hat{\psi}(n) R_{0}^{-n}(f)$ belongs to $\mathcal{B}$ and by (15), it is equal to $\mathcal{C}(\psi f)$. This finishes the proof.

Let $I$ be a closed ideal of $\mathcal{B}$ and $g \in \mathcal{B}$. We denote by $I(g)$ the division ideal of $I$ by $g$, that is

$$
I(g)=\{f \in \mathcal{B}: f g \in I\} .
$$

Clearly $I(g)$ is a closed ideal of $\mathcal{B}$ that contains $I$.

The following lemma is known in the case $\mathcal{B}=A^{+}(\mathbb{T})$ (see Lemma 1.2 and Lemma 1.5 of $[7]$ ). The proof is based on a similar argument used 
in [7] and on Lemma 2.9 established below. We include the proof to see how Lemma 2.9 operates here.

Lemma 2.10. Suppose that $\mathcal{B}$ satisfies conditions (H1)-(H3). Let I be a closed ideal of $\mathcal{B}$ such that $h^{0}(I)$ is at most countable and let $g$ be an element of $U_{I} H^{\infty} \cap \mathcal{B}$. Then $U_{I(g)}=1$.

Proof: Notice that $I \subset I(g)$, which implies that $h^{0}(I(g)) \subset h^{0}(I)$. Let $\lambda \in h^{0}(I) \cap \mathbb{D}$. Let $f \in I$ such that $k_{f}(\lambda)=k_{I}(\lambda)$. Define

$$
\tilde{f}(z)=\left\{\begin{array}{ll}
\frac{f(z)}{(z-\lambda)^{k_{I}(\lambda)+1}} & \text { if } z \in \overline{\mathbb{D}}, z \neq \lambda \\
\frac{f^{\left(k_{I}(\lambda)+1\right)}(\lambda)}{\left(k_{I}(\lambda)+1\right) !} & \text { if } z=\lambda
\end{array} .\right.
$$

We have $\tilde{f}=R_{\lambda}{ }^{k_{I}(\lambda)+1}(f)$. By Lemma 2.1, $\tilde{f} \in \mathcal{B}$. Since $k_{g}(\lambda) \geq k_{I}(\lambda)$, we have $\tilde{f} g=f h$, with $h=R_{\lambda}{ }^{k_{I}(\lambda)+1}(g) \in \mathcal{B}$. Hence $\tilde{f} g \in I$, which means that $\tilde{f} \in I(g)$. Now, by construction, $\tilde{f}(\lambda) \neq 0$, which proves that $\lambda \notin h^{0}(I(g))$. This shows that

$$
h^{0}(I(g)) \subset h^{0}(I) \cap \mathbb{T}
$$

and consequently, $B_{I(g)}=1$. Since $h^{0}(I)$ is at most countable, it suffices to show that $\mu_{I(g)}(\{z\})=0$ for all $z \in h^{0}(I) \cap \mathbb{T}$, to prove that $S_{I(g)}=1$. Let $z_{0} \in h^{0}(I) \cap \mathbb{T}$ and let $\varphi$ be a nonzero outer function in $a^{\infty}(\mathbb{D}) \subset \mathcal{B}$ such that $\varphi^{(k)}\left(z_{0}\right)=0, k \geq 0$. Let $\psi(z)=\varphi(z) \overline{S(z)}, z \in \mathbb{T}$, where $S(z)=\exp \left(\mu_{I}\left(\left\{z_{0}\right\}\right) \frac{z+z_{0}}{z-z_{0}}\right)$. It is easily seen, by Taylor formulae, that $\psi$ is infinitely differentiable on $\mathbb{T}$.

Let $f \in I$. Since $\mu(f) \geq \mu_{I} \geq \mu_{I}\left(\left\{z_{0}\right\}\right) \delta_{z_{0}}, \delta_{z_{0}}$ being the Dirac measure at $z_{0}$, function $\varphi f / S \in a(\mathbb{D})$ and coincides in $\mathbb{D}$ with $\mathcal{C}(\psi f)$. Similarly we have $\varphi g / S \in A(\mathbb{D})$ and $\varphi g / S=\mathcal{C}(\psi g)$ in $\mathbb{D}$. By Lemma 2.9, $\mathcal{C}(\psi f)$ and $\mathcal{C}(\psi g)$ belong to $\mathcal{B}$. Hence $\mathcal{C}(\psi g) f \in I$. Thus $\mathcal{C}(\psi f) g=$ $\mathcal{C}(\psi g) f \in I$, which means that $\mathcal{C}(\psi f) \in I(g)$.

Now since $\mathcal{C}(\psi f)=\varphi f / S$, we see that

$$
\mu_{I(g)}\left(\left\{z_{0}\right\}\right) \leq \mu_{(\mathcal{C}(\psi f))}\left(\left\{z_{0}\right\}\right)=\mu_{f}\left(\left\{z_{0}\right\}\right)-\mu_{I}\left(\left\{z_{0}\right\}\right) \leq\left\|\mu_{f}-\mu_{I}\right\| .
$$

By Lemma 2.6 we obtain that $\mu_{I(g)}\left(\left\{z_{0}\right\}\right)=0$, which finishes the proof.

Let $U$ be an inner function and $E_{0}, \ldots, E_{N_{\mathcal{B}}}$ be closed subsets of $\mathbb{T}$ such that $E_{N_{\mathcal{B}}} \subset \cdots \subset E_{0}$. We set

$I\left(U ; E_{0}, \ldots, E_{N_{\mathcal{B}}}\right)=\left\{f \in \mathcal{B}: U \mid U(f)\right.$ and $f^{(k)}=0$ on $\left.E_{k} \quad\left(0 \leq k \leq N_{\mathcal{B}}\right)\right\}$. 
Clearly $I\left(U ; E_{0}, \ldots, E_{N_{\mathcal{B}}}\right)$ is a closed ideal of $\mathcal{B}$. The following theorem is the main result of this paper. We recall that $\mathcal{B}$ is a semi-simple unital commutative Banach algebra of functions holomorphics in $\mathbb{D}$ and continuous on $\overline{\mathbb{D}}$, endowed with pointwise product and continuously imbedded in $a(\mathbb{D})$.

Theorem 2.11. Assume that $\mathcal{B}$ satisfies conditions (H1)-(H3) and the analytic Ditkin condition. Let $I$ be a closed ideal of $\mathcal{B}$ such that $h^{0}(I)$ is at most countable. Then $I$ is standard.

Proof: We have obviously $I \subset I_{0}$, where $I_{0}=I\left(U_{I} ; h^{0}(I) \cap \mathbb{T}, \ldots\right.$, $\left.h^{N_{\mathcal{B}}}(I) \cap \mathbb{T}\right)$. Let $g \in I_{0}$. To prove that $g \in I$, we are going to prove that $I(g)=\mathcal{B}$. Notice that since $I \subset I(g)$, we have $h^{0}(I(g)) \subset h^{0}(I)$. By Lemma 2.10, we have $U_{I(g)}=1$ and so

$$
h^{0}(I(g)) \subset h^{0}(I) \cap \mathbb{T} .
$$

Let $z_{0} \in\left(h^{0}(I) \backslash h^{N_{\mathcal{B}}}(I)\right) \cap \mathbb{T}$. Then, $z_{0}$ is an isolated point of $h^{0}(I)$. So, by the idempotent Shilov theorem (see [10]), there exists $\psi \in \mathcal{B}$ such that

$$
\left\{\begin{array}{l}
\psi\left(z_{0}\right)=1 \\
\psi=0
\end{array} \quad \text { on } h^{0}(I) \backslash\left\{z_{0}\right\} \quad \text { and } \quad \psi(1-\psi) \in I .\right.
$$

As $I \subset I(\psi)$, we have, for all $k \in\left\{0, \ldots, N_{\mathcal{B}}\right\}, h^{k}(I(\psi)) \subset h^{k}(I)$. Moreover, since $1-\psi \in I(\psi)$ and $1 \notin I(\psi)$, we have $h^{0}(I(\psi))=\left\{z_{0}\right\}$. And, as $z_{0} \notin h^{N_{\mathcal{B}}}(I), h^{N_{\mathcal{B}}}(I(\psi))=\emptyset$. So we deduce from Lemma 2.8 that $U_{I(\psi)}=1$. Consequently, we deduce from Lemma 2.7 that

$$
I(\psi)=\left\{f \in \mathcal{B}: f^{(k)}\left(z_{0}\right)=0 \quad\left(0 \leq k \leq k_{I(\psi)}\left(z_{0}\right)\right)\right\} .
$$

Since $k_{g}\left(z_{0}\right) \geq k_{I}\left(z_{0}\right) \geq k_{I(\psi)}\left(z_{0}\right)$, we get in particular that $g \in I(\psi)$. This proves that $\psi \in I(g)$, so that $z_{0} \notin h^{0}(I(g))$. Therefore, we have proved that

$$
h^{0}(I(g)) \subset h^{N_{\mathcal{B}}}(I) .
$$

Now, suppose that $h^{0}(I(g)) \neq \emptyset$. Since $h^{0}(I(g))$ is at most countable, there exists $z_{0}$ an isolated point of $h^{0}(I(g))$. Using once more the idempotent Shilov Theorem, there exists $\varphi \in \mathcal{B}$ such that

$$
\left\{\begin{array}{l}
\varphi\left(z_{0}\right)=1 \\
\varphi=0
\end{array} \quad \text { on } h^{0}(I(g)) \backslash\left\{z_{0}\right\} \quad \text { and } \quad \varphi(1-\varphi) \in I(g) .\right.
$$

Let $J:=(I(g))(\varphi)$ be the division ideal of $I(g)$ by $\varphi$. As $z_{0} \in h^{N_{\mathcal{B}}}(I)$, $g$ vanishes with all its derivatives (of order less or equal than $N_{\mathcal{B}}$ ) at $z_{0}$. And since $\mathcal{B}$ satisfies the analytic Ditkin condition, we deduce from 
Lemma 2.5 that there exists a sequence $\left(\sigma_{n}\right)_{n>0}$ included in $\mathcal{B}$ such that $m_{\sigma_{n}}\left(z_{0}\right) \geq N_{\mathcal{B}}+1$ and

$$
\lim _{n \rightarrow+\infty}\left\|\sigma_{n} \varphi g-\varphi g\right\|_{\mathcal{B}}=0 .
$$

Now, since $I(g) \subset J$, we deduce from Lemma 2.10 that $U_{J}=1$. Moreover, since $1-\varphi \in J$, we have $h(J) \subset\left\{z_{0}\right\}$. Then, Lemma 2.7 gives the characterisation of $J$. In particular, by Lemma 2.4, $\sigma_{n} \in J, n \geq 0$. This means that, for all $n \geq 0, \sigma_{n} \varphi g \in I$. Hence, since $I$ is closed, we deduce from (16) that $\varphi g \in I$. In other words, $\varphi \in I(g)$, which is in contradiction with the fact that $z_{0} \in h^{0}(I(g))$. Finally, we have proved that $h^{0}(I(g))=\emptyset$, that is $I(g)=\mathcal{B}$.

Let $\mathcal{H}$ be a Banach algebra of continuous functions on $\mathbb{T}$. We say that $\mathcal{H}$ is an homogeneous Banach algebra if it satisfies the following conditions:

(1) $\mathcal{H}$ is a semi-simple and commutative Banach algebra with maximal ideal space $\mathbb{T}$.

(2) The set of trigonometric polynomials is a dense subset of $\mathcal{H}$.

(3) For all $f \in \mathcal{H}$ and $\tau \in \mathbb{R}$, we have $\left\|f_{\tau}\right\|_{\mathcal{H}}=\|f\|_{\mathcal{H}}$, where $f_{\tau}\left(e^{i t}\right)=$ $f\left(e^{i(t-\tau)}\right)$.

Let $\mathcal{H}$ be an homogeneous algebra on $\mathbb{T}$. We denote by $\mathcal{H}^{+}$the closed subalgebra of $\mathcal{H}$ generated by $\left\{e^{i n t}: n \geq 0\right\}$.

Notice that conditions (2) and (3) implies that for every $\tau_{0} \in \mathbb{R}$, $\left\|f_{\tau}-f_{\tau_{0}}\right\|_{\mathcal{H}} \rightarrow 0$, as $\tau \rightarrow \tau_{0}$. It follows then from Theorem 2.12 of [16] that $\mathcal{H}^{+}$consists of functions in $\mathcal{H}$ having analytic extension into $\mathbb{D}$. Then, the maximal ideal space of $\mathcal{H}^{+}$is $\overline{\mathbb{D}}$.

Recall that we denote by $\mathcal{C}^{n}(\mathbb{T})$ the space of $n$ times continuously differentiable functions on $\mathbb{T}$ and we set $\mathcal{C}^{\infty}(\mathbb{T})=\bigcap_{n>0} \mathcal{C}^{n}(\mathbb{T})$.

Suppose that $\mathcal{H}$ contains $\mathcal{C}^{\infty}(\mathbb{T})$, which implies in particular that $\mathcal{H}$ is a regular algebra. Also this implies that $\mathcal{C}^{k}(\mathbb{T}) \subset \mathcal{H}$ for some nonnegative integer $k$. It follows that $N_{\mathcal{H}}$, the greatest of the integers $n$ for which $\mathcal{H} \subset \mathcal{C}^{n}(\mathbb{T})$, is bounded. We say that $\mathcal{H}$ satisfies the Ditkin condition if for each function $f \in \mathcal{H}$ such that $f^{(k)}(1)=0,0 \leq k \leq N_{\mathcal{H}}$, there exists a sequence $\left(v_{n}\right)_{n \geq 0}$ in $\mathcal{H}$ satisfying the following properties:

(1) For all $n \geq 0, v_{n}=0$ on a neighbourhood of 1 .

(2) $\lim _{n \rightarrow+\infty}\left\|\left(1-v_{n}\right) f\right\|_{\mathcal{H}}=0$.

We say that $\mathcal{H}$ satisfies the analytic Ditkin condition if $\mathcal{H}^{+}$satisfies the analytic Ditkin condition. 
Corollary 2.12. Let $\mathcal{H}$ be an homogeneous Banach algebra on $\mathbb{T}$ that contains $\mathcal{C}^{\infty}(\mathbb{T})$ and satisfies the analytic Ditkin condition. Let $I$ be a closed ideal of $\mathcal{H}^{+}$such that $h^{0}(I)$ is at most countable. Then $I$ is standard.

Proof: By Theorem 2.11 it suffices to check that $\mathcal{H}^{+}$satisfies conditions (H1)-(H3). This is clearly the case for $(\mathrm{H} 1)$ and $(\mathrm{H} 2)$. It remains to check condition (H3). As we have observed before there exists a nonnegative integer $k$ such that $\mathcal{C}^{k}(\mathbb{T}) \subset \mathcal{H}$. By the closed Graph theorem this imbedding is continuous. So there exists a constant $C$ such that

$$
\|f\|_{\mathcal{B}} \leq C\|f\|_{\mathcal{C}^{k}(\mathbb{T})}, \quad f \in \mathcal{C}^{k}(\mathbb{T}) .
$$

In particular we have $\left\|\alpha^{n}\right\|=O\left(|n|^{k}\right),|n| \rightarrow \infty$. So there exists a constant $C$ such that for every $\lambda \in \mathbb{D}$,

$$
\left\|(\alpha-\lambda)^{-1}\right\|_{\mathcal{H}} \leq \sum_{n \geq 0}\left\|\alpha^{-n-1}\right\|_{\mathcal{H}}|\lambda|^{n} \leq C(|1-| \lambda \mid)^{-k-1} .
$$

So, for every $\lambda \in \mathbb{D}$ and $f \in \mathcal{H}^{+}, R_{\lambda}(f) \in \mathcal{H}^{+}$and

$$
\left\|R_{\lambda}(f)\right\|_{\mathcal{H}} \leq\left\|(\alpha-\lambda)^{-1}\right\|_{\mathcal{H}}\|f-f(\lambda)\|_{\mathcal{H}} \leq 2 C(|1-| \lambda \mid)^{-k-1}\|f\|_{\mathcal{H}} .
$$

It follows now from Lemma 2.1 that $\mathcal{H}^{+}$satisfy condition (H3).

Remark 2.13. 1) Let $\mathcal{H}$ be an homogeneous Banach algebra on $\mathbb{T}$ that contains $\mathcal{C}^{\infty}(\mathbb{T})$ and such that $\mathcal{H}^{+}$satisfies the analytic Ditkin condition. It is announced in $[\mathbf{3}]$ that if $I$ is a closed ideal of $\mathcal{H}^{+}$such that $h^{0}(I)$ is a finite set then

$$
I=I^{\mathcal{H}} \cap U_{I} H^{\infty}(\mathbb{D}),
$$

where $I^{\mathcal{H}}$ is the closed ideal of $\mathcal{H}$ generated by $I$.

2) Let $\mathcal{H}$ be an homogeneous Banach algebra on $\mathbb{T}$ such that

$$
\left\|\alpha^{n}\right\|_{\mathcal{H}}=O\left(n^{k}\right)(n \rightarrow+\infty), \text { for some } k \geq 0,
$$

and

$$
\lim _{n \rightarrow+\infty} \frac{\log \left\|\alpha^{-n}\right\|_{\mathcal{H}}}{\sqrt{n}}=0 .
$$

We can show that if $I$ is a closed ideal of $\mathcal{H}$ such that $\tilde{h}^{0}(I)$ is countable then

$$
I=\left\{f \in \mathcal{H}: f^{(k)}=0 \text { on } \tilde{h}^{k}(I), \quad\left(0 \leq k \leq N_{\mathcal{H}}\right)\right\},
$$

where $\tilde{h}^{k}(I)=\left\{z \in \mathbb{T}: f(z)=\cdots=f^{(k)}(z)=0,(f \in I)\right\}$. The proof of this result uses similar arguments as in the case of Theorem 2.11 with some simplications, since there is no problem in this case with the inner factor. 


\section{Closed ideals in $\ell^{1}(\omega)$ and $L^{1}\left(\mathbb{R}^{+}, \omega\right)$}

Let $s$ be a nonnegative real number. We set $\omega_{s}(t)=(1+t)^{s}, t \geq 0$. Denote by $\ell^{1}\left(\omega_{s}\right)$ the set of all complex sequences $x=\left(x_{n}\right)_{n \geq 0}$ such that

$$
\left\|x_{n}\right\|_{s}:=\sum_{n \geq 0}\left|x_{n}\right| \omega_{s}(n)<+\infty .
$$

Clearly $\ell^{1}\left(\omega_{s}\right)$, equipped with convolution product and norm \|\|$_{s}$, is a semi-simple unitary commutative Banach algebra. The set of maximal ideals of $\ell^{1}\left(\omega_{s}\right)$ can be identified with $\overline{\mathbb{D}}$. Considering the Gelfand transfom $x \rightarrow \hat{x}$, where $\hat{x}(z)=\sum_{n>0} x_{n} z^{n}, z \in \overline{\mathbb{D}}$, we see that $\ell^{1}\left(\omega_{s}\right)$ is isometrically isomorphic to the Beurling weighted Banach algebra

$$
A_{s}^{+}(\mathbb{T})=\left\{f \in a(\mathbb{D}):\|f\|_{s}:=\sum_{n \geq 0}|\hat{f}(n)| \omega_{s}(n)<+\infty\right\} .
$$

So we will just be interested by closed ideals of $A_{s}^{+}(\mathbb{T})$. The structure of closed ideals $I$ of $A_{s}^{+}(\mathbb{T})$ such that $h^{0}(I)$ is countable, is known only in the cases when $s<1$ (see [23], [3] and [7]) and when $s \geq 1$ and $U_{I}=1$ (see $[\mathbf{1}])$. Here we give a complete characterisation of such ideals.

With notation of the above section, we have $A_{s}^{+}(\mathbb{T})=\left(A_{s}(\mathbb{T})\right)^{+}$where

$$
A_{s}(\mathbb{T})=\left\{f \in \mathcal{C}(\mathbb{T}):\|f\|_{s}:=\sum_{n \in \mathbb{Z}}|\hat{f}(n)|(1+|n|)^{s}<+\infty\right\} .
$$

Clearly $A_{s}(\mathbb{T})$ is an homogeneous Banach algebra on $\mathbb{T}$ that contain $\mathcal{C}^{\infty}(\mathbb{T})$. Moreover by Proposition 2.4 of $[\mathbf{1}], A_{s}^{+}(\mathbb{T})$ satisfies the analytic Ditkin condition. Then we deduce immediately from Corollary 2.12 the following result.

Theorem 3.1. Let $s$ be a nonnegative real number, and $I$ be a closed ideal of $A_{s}^{+}(\mathbb{T})$ such that $h^{0}(I)$ is at most countable. Then I is standard.

Now we turn to the Banach algebra

$L^{1}\left(\mathbb{R}^{+}, \omega_{s}\right)=\left\{f\right.$ measurable on $\left.\mathbb{R}^{+}:\|f\|_{1, s}=\int_{0}^{+\infty}|f(t)| \omega_{s}(t) \mathrm{d} t<+\infty\right\}$.

$\left(L^{1}\left(\mathbb{R}^{+}, \omega_{s}\right),\|\|_{1, s}\right)$, equipped with the convolution product, is a Banach algebra. We denote by $P$ the open half plane $\{z \in \mathbb{C}: \operatorname{Re}(z)>0\}$. For $f \in L^{1}\left(\mathbb{R}^{+}\right)$, we define the Laplace transform $\mathcal{L}(f)$ by

$$
\mathcal{L}(f)(z)=\int_{0}^{+\infty} f(t) e^{-t z} \mathrm{~d} t, \quad(z \in \bar{P}) .
$$


Let $J$ be a closed ideal of $L^{1}\left(\mathbb{R}^{+}, \omega_{s}\right)$. We set

$h^{k}(J)=\left\{z \in \bar{P}: \mathcal{L}(f)(z)=\cdots=\mathcal{L}(f)^{(k)}(z)=0, \quad(f \in J)\right\}, \quad(0 \leq k \leq[s])$,

where $[s]$ is the integer such that $[s] \leq s<[s]+1$. And we denote by $T_{J}$ its inner factor, that is the greatest common divisor of all Laplace transform of nonzero functions in $J$. Also we set $\beta_{J}=\inf _{f \in J} \beta_{f}$, where

$$
\beta_{f}=\sup \{a: f=0 \text { on }[0, a] \text { (almost everywhere) }\} .
$$

Notice that $J=L^{1}\left(\mathbb{R}^{+}, \omega_{s}\right)$ if and only if $h^{0}(I)=\emptyset$ and $\beta_{J}=0([\mathbf{2 2}]$, [11]). We say that $J$ is modular if $L^{1}\left(\mathbb{R}^{+}, \omega_{s}\right) / J$ admits a unit.

It is well-known that $J$ is a closed modular ideal of $L^{1}\left(\mathbb{R}^{+}, \omega_{s}\right)$ if and only if $h^{0}(J)$ is compact and $\beta_{J}=0$ (in case $s=0$, see for example [4, Proposition 2.1]). When $s=0$, V. P. Gurarii showed in [11] that if $J$ is a closed ideal of $L^{1}\left(\mathbb{R}^{+}\right)=L^{1}\left(\mathbb{R}^{+}, \omega_{0}\right)$ such that $h^{0}(J)$ is at most countable, then

$$
J=\mathcal{L}^{-1}\left(\mathcal{L}(J)^{\infty}\right)
$$

where $\mathcal{L}(J)^{\infty}$ is the closure of $\mathcal{L}(J)$ in $A_{0}(P)$, the algebra of all functions $f$ which are continuous on $\bar{P}$, holomorphic in $P$, and such that $f(z) \rightarrow 0$ as $|z| \rightarrow+\infty$. The closed ideals of $A_{0}(P)$ are given by the Beurling-Rudin theorem, thanks to a conformal transform between $P$ and $\mathbb{D}$. So, if $h^{0}(J)$ is at most countable, we have

$$
J=\left\{f \in L^{1}\left(\mathbb{R}^{+}\right): T_{J} \mid \mathcal{L}(f) \text { and } \mathcal{L}(f)=0 \text { on } h^{0}(J) \cap i \mathbb{R}\right\} .
$$

Using a result of $\mathrm{H}$. Hedenmalm, we will deduce from Theorem 3.1 a similar result concerning closed ideals of $L^{1}\left(\mathbb{R}^{+}, \omega_{s}\right)$, for any nonnegative real number $s$.

If $I$ is a closed ideal of $A_{s}^{+}(\mathbb{T})$, we denote by $\pi=\pi_{I}$ the canonical surjection from $A_{s}^{+}(\mathbb{T})$ onto $A_{s}^{+}(\mathbb{T}) / I$. If $h^{0}(I) \subset \overline{\mathbb{D}} \backslash[-1,0]$, we can define the function $z \mapsto \pi(\alpha)^{z}$, which is an entire function of finite exponential type. Furthermore, we have

$$
\begin{aligned}
\left\|\pi(\alpha)^{t}\right\|_{s} & \leq \sup _{0 \leq u \leq 1}\left\|\pi(\alpha)^{u}\right\|_{s}(1+[t])^{s} \\
& \leq \sup _{0 \leq u \leq 1}\left\|\pi(\alpha)^{u}\right\|_{s}(1+t)^{s} \quad(t \geq 0) .
\end{aligned}
$$

Then, we define $\Phi_{I}: L^{1}\left(\mathbb{R}^{+}, s\right) \rightarrow A_{s}^{+}(\mathbb{T}) / I$ by

$$
\Phi_{I}(f)=\int_{0}^{+\infty} f(t) \pi(\alpha)^{t} \mathrm{~d} t
$$


$\Phi_{I}$ is a continuous homomorphism. We set

$$
\mathcal{I}=\left\{I \text { closed ideals of } A_{s}^{+}(\mathbb{T}): h^{0}(I) \subset \overline{\mathbb{D}} \backslash[-1,0]\right\}
$$

and

$\mathcal{J}=\left\{J\right.$ closed modular ideals of $\left.L^{1}\left(\mathbb{R}^{+}, s\right): h^{0}(J) \subset[0,+\infty) \times(-\pi, \pi)\right\}$.

The following result, due to H. Hedenmalm ([13, Theorem 4.3 and Remark 4.5]), is essential for our purpose, since it gives a one-to-one correspondence between $\mathcal{I}$ and $\mathcal{J}$ (see also [4, Theorem 2.2]).

Theorem $3.2([\mathbf{1 3}])$. The mapping $\Theta: I \mapsto \operatorname{ker} \Phi_{I}$ defines a bijection between $\mathcal{I}$ and $\mathcal{J}$. Futhermore, if $J \in \mathcal{J}$, we have

$$
\Theta^{-1}(J)=\left\{f \in A_{s}^{+}(\mathbb{T}):\left(\sum_{n=0}^{+\infty} \widehat{f}(n) \delta_{n}\right) * g \in J, \quad\left(g \in L^{1}\left(\mathbb{R}^{+}, \omega_{s}\right)\right)\right\},
$$

where $\delta_{n}$ is the Dirac measure at $n$.

Until the end of this paper, $X^{*}$ will denote the dual of a normed space $X$, and the duality will be denoted by

$$
\langle f, g\rangle \quad\left(f \in X, g \in X^{*}\right) .
$$

Let $I$ be a closed ideal of $A_{s}^{+}(\mathbb{T})$ and $\pi=\pi_{I}$ the canonical surjection from $A_{s}^{+}(\mathbb{T})$ onto $A_{s}^{+}(\mathbb{T}) / I$. Let $0 \leq k \leq[s]$ and $w \in h^{k}(I)$. The functional $\chi_{w}^{(k)}: g \mapsto g^{(k)}(w)$ is continuous on $A_{s}^{+}(\mathbb{T})$ and we have $I \subset$ $\operatorname{ker} \chi_{w}^{(k)}$. So there exists $\tilde{\chi}_{w}^{(k)} \in\left(A_{s}^{+}(\mathbb{T}) / I\right)^{*}$ such that

$$
\left\langle\pi(g), \tilde{\chi}_{w}^{(k)}\right\rangle=\left\langle g, \chi_{w}^{(k)}\right\rangle \quad\left(g \in A_{s}^{+}(\mathbb{T})\right) .
$$

Let $\Psi$ be an analytic function on a neighbourhood of $h^{0}(I)$. We use the Dunford-Schwarz functional calculus to define $\Psi(\pi(\alpha))$ by

$$
\Psi(\pi(\alpha))=\frac{1}{2 i \pi} \int_{\partial \Omega} \Psi(\xi)(\xi-\pi(\alpha))^{-1} \mathrm{~d} \xi,
$$

where $\partial \Omega$ is the boundary of a suitable open neighborhood $\Omega$ of $h^{0}(I)$ in which $\Psi$ is holomorphic. Then, we have the following lemma.

Lemma 3.3. Let $I$ be a closed ideal of $A_{s}^{+}(\mathbb{T})$. Let $\Psi$ be an analytic function on a neighbourhood of $h^{0}(I)$. Then

$$
\left\langle\Psi(\pi(\alpha)), \tilde{\chi}_{w}^{(k)}\right\rangle=\Psi^{(k)}(w) \quad\left(0 \leq k \leq[s], w \in h^{k}(I)\right) .
$$

Proof: We have

$$
\Psi(\pi(\alpha))=\frac{1}{2 i \pi} \int_{\partial \Omega} \Psi(\xi)(\xi-\pi(\alpha))^{-1} \mathrm{~d} \xi .
$$


Since $\tilde{\chi}_{w}^{(k)}$ is a continuous functional, we have

$$
\left\langle\Psi(\pi(\alpha)), \tilde{\chi}_{w}^{(k)}\right\rangle=\frac{1}{2 i \pi} \int_{\partial \Omega} \Psi(\xi)\left\langle(\xi-\pi(\alpha))^{-1}, \tilde{\chi}_{w}^{(k)}\right\rangle \mathrm{d} \xi .
$$

Hence, the result will follow from the Cauchy integral formulae, if we prove that

$$
\left\langle(\xi-\pi(\alpha))^{-1}, \tilde{\chi}_{w}^{(k)}\right\rangle=k !(\xi-w)^{-k-1} .
$$

Let $\xi \in \partial \Omega$ and $g \in A_{s}^{+}(\mathbb{T})$ such that $\pi(g)(\xi-\pi(\alpha))=1$. Since, $w \in h^{k}(I)$, we have the following relations:

$$
\begin{array}{ll} 
& g(w)(\xi-w)=1 \\
\text { and } \quad g^{(j)}(w)(\xi-w)-j g^{(j-1)}(w)=0, \quad(1 \leq j \leq k) .
\end{array}
$$

So, we deduce easily from these equalities that

$$
g^{(k)}(w)=k !(\xi-w)^{-k-1} .
$$

Then, we have

$$
\begin{aligned}
\left\langle(\xi-\pi(\alpha))^{-1}, \tilde{\chi}_{w}^{(k)}\right\rangle & =\left\langle\pi(g), \tilde{\chi}_{w}^{(k)}\right\rangle \\
& =g^{(k)}(w) \\
& =k !(\xi-w)^{-k-1} .
\end{aligned}
$$

Then the result follows from the above equality, (18) and the Cauchy integral formulae.

We also need the two following lemmas.

Lemma 3.4. Let $I \in \mathcal{I}$ and $J=\Theta(I)$. Then, we have

$$
h^{k}(I)=e^{-h^{k}(J)}, \quad(0 \leq k \leq[s]) .
$$

Proof: Let $0 \leq k \leq[s], w \in h^{k}(I)$ and $f \in J$. We have $\Phi_{I}(f)=0$ and in particular,

$$
\left\langle\int_{0}^{+\infty} f(t) \pi(\alpha)^{t} \mathrm{~d} t, \tilde{\chi}_{w}^{(j)}\right\rangle=0 \quad(0 \leq j \leq k) .
$$

Now, applying Lemma 3.3 with $\Psi(z)=z^{t}(t \geq 0)$, we get, for all $0 \leq$ $j \leq k$,

$$
\left\langle\int_{0}^{+\infty} f(t) \pi(\alpha)^{t} \mathrm{~d} t, \tilde{\chi}_{w}^{(j)}\right\rangle=\int_{0}^{+\infty} f(t)\left\langle\pi(\alpha)^{t}, \tilde{\chi}_{w}^{(j)}\right\rangle \mathrm{d} t
$$

$$
=\int_{0}^{+\infty} f(t) t(t-1) \ldots(t-j+1) w^{t-j} \mathrm{~d} t,
$$


with the understanding that $t(t-1) \ldots(t-j-1)=1$ if $j=0$. So, we easily deduce from (19) and (20) that, for all $0 \leq j \leq k$,

$$
\mathcal{L}(f)^{(j)}(-\log w)=(-1)^{j} \int_{0}^{+\infty} f(t) t^{j} w^{t} \mathrm{~d} t=0 .
$$

This proves that $-\log w \in h^{k}(J)$, and so

$$
h^{k}(I) \subset e^{-h^{k}(J)} .
$$

Now, let $w \in h^{k}(J)$ and $f \in I$. For $g \in L^{1}\left(\mathbb{R}^{+}, \omega_{s}\right)$ we set $F_{g}=$ $\left(\sum_{n=0}^{+\infty} \widehat{f}(n) \delta_{n}\right) * g$. We have

$$
\begin{aligned}
\mathcal{L}\left(F_{g}\right)(z) & =\sum_{n=0}^{+\infty} \widehat{f}(n) \int_{0}^{+\infty}\left(\delta_{n} * g\right)(t) e^{-t z} \mathrm{~d} t \\
& =f\left(e^{-z}\right) \mathcal{L}(g)(z), \quad\left(z \in \bar{P}, g \in L^{1}\left(\mathbb{R}^{+}, \omega_{s}\right)\right) .
\end{aligned}
$$

According to Theorem 3.2, we have $F_{g} \in J$, for all $g \in L^{1}\left(\mathbb{R}^{+}, \omega_{s}\right)$. It follows that

$$
\mathcal{L}\left(F_{g}\right)^{(j)}(w)=0, \quad\left(0 \leq j \leq k, g \in L^{1}\left(\mathbb{R}^{+}, \omega_{s}\right)\right) .
$$

Now, we easily deduce from (21) and (22) that

$$
f^{(j)}\left(e^{-w}\right)=0, \quad(0 \leq j \leq k) .
$$

This proves that

$$
e^{-h^{k}(J)} \subset h^{k}(I),
$$

and concludes the proof of this lemma.

Lemma 3.5. Let $J, J^{\prime} \in \mathcal{J}$. Then, we have

$$
T_{J}=T_{J^{\prime}} \Longleftrightarrow U_{\Theta^{-1}(J)}=U_{\Theta^{-1}\left(J^{\prime}\right)} .
$$

Proof: Let $J \in \mathcal{J}$ and set $I=\Theta^{-1}(J)$. We begin to prove that

$$
J_{0}=\Theta_{0}\left(I_{0}\right) \text {, }
$$

where $J_{0}$ (resp. $I_{0}$ ) is the closure of $J$ in $L^{1}\left(\mathbb{R}^{+}\right)\left(\right.$resp. $\left.A^{+}(\mathbb{T})\right)$ and where $\Theta_{0}$ is the map $\Theta$ corresponding to the case $s=0$. Clearly $J_{0}$ and $I_{0}$ are closed ideals. Moreover we have $T_{J_{0}}=T_{J}$ and $U_{I_{0}}=U_{I}$.

By Theorem 3.2 we have

$$
I=\left\{f \in A_{s}^{+}(\mathbb{T}):\left(\sum_{n=0}^{+\infty} \widehat{f}(n) \delta_{n}\right) * g \in J, \quad\left(g \in L^{1}\left(\mathbb{R}^{+}, \omega_{s}\right)\right)\right\} .
$$


Since, for every $g \in L^{1}\left(\mathbb{R}^{+}, \omega_{s}\right)$, the map $f \rightarrow\left(\sum_{n=0}^{+\infty} \widehat{f}(n) \delta_{n}\right) * g$ is continuous from $A^{+}(\mathbb{T})$ in $L^{1}\left(\mathbb{R}^{+}\right)$, we get

$$
I_{0} \subset\left\{f \in A^{+}(\mathbb{T}):\left(\sum_{n=0}^{+\infty} \widehat{f}(n) \delta_{n}\right) * g \in J_{0}, \quad\left(g \in L^{1}\left(\mathbb{R}^{+}, \omega_{s}\right)\right)\right\} .
$$

Also since, for every $f \in A^{+}(\mathbb{T})$, the map $g \rightarrow\left(\sum_{n=0}^{+\infty} \widehat{f}(n) \delta_{n}\right) * g$ is continuous from $L^{1}\left(\mathbb{R}^{+}\right)$in itself and since $L^{1}\left(\mathbb{R}^{+}, \omega_{s}\right)$ is dense in $L^{1}\left(\mathbb{R}^{+}\right)$, we obtain

$$
I_{0} \subset\left\{f \in A^{+}(\mathbb{T}):\left(\sum_{n=0}^{+\infty} \widehat{f}(n) \delta_{n}\right) * g \in J_{0}, \quad\left(g \in L^{1}\left(\mathbb{R}^{+}\right)\right)\right\} .
$$

This means that $I_{0} \subset \Theta_{0}^{-1}\left(J_{0}\right)$. Since $\Theta_{0}$ is increasing with respect to inclusion order we have

$$
\Theta_{0}\left(I_{0}\right) \subset J_{0}
$$

It remains to prove the other inclusion. Denote by $\psi$ the homomorphism from $A_{s}^{+}(\mathbb{T}) / I$ into $A^{+}(\mathbb{T}) / I_{0}$ defined by $\psi\left(\pi_{I}(f)\right)=\pi_{I_{0}}(f)$. Since $\psi$ is an homomorphism, it is easily seen that

$$
\Phi_{\left.I_{0}\right|_{A_{s}^{+}(\mathbb{T})} ^{0}}^{0}=\psi \circ \Phi_{I}
$$

where $\Phi_{I_{0}}^{0}$ is the map $\Phi_{I}$ corresponding to $s=0$ and $I=I_{0}$. Hence, we have $\operatorname{ker} \Phi_{I} \subset \operatorname{ker} \Phi_{I_{0}}^{0} \cap A_{s}^{+}(\mathbb{T}) \subset \operatorname{ker} \Phi_{I_{0}}^{0}$. In other words, we have $J \subset \Theta_{0}\left(I_{0}\right)$. Since $\Theta_{0}\left(I_{0}\right)$ is closed in $L^{1}\left(\mathbb{R}^{+}\right)$, we get

$$
J_{0} \subset \Theta_{0}\left(I_{0}\right) .
$$

Finally, we have proved (23).

Let $J^{\prime} \in \mathcal{J}, I^{\prime}=\Theta^{-1}\left(J^{\prime}\right)$ and $J_{0}^{\prime}$ (resp. $I_{0}^{\prime}$ ) be the closure of $J^{\prime}$ (resp. $\left.I^{\prime}\right)$ in $L^{1}\left(\mathbb{R}^{+}\right)$(resp. $A^{+}(\mathbb{T})$ ). We have $J_{0}^{\prime}=\Theta_{0}\left(I_{0}\right)$.

Denote by $H^{\infty}(\mathbb{D})$ (resp. $H^{\infty}(P)$ ) the space of bounded holomorphic function on $\mathbb{D}$ (resp. $P$ ). Denote also by $A_{0}(P)$ the space of holomorphic functions on $P$, continuous on $\bar{P}$, and vanishing at infity.

It follows from Lemma 3.7 of [4] and from (23) that

$$
\Theta_{0}\left(U_{I_{0}} H^{\infty}(\mathbb{D}) \cap A^{+}(\mathbb{T})\right)=\mathcal{L}^{-1}\left(T_{J_{0}} H^{\infty}(P) \cap A_{0}(P)\right)
$$

and

$$
\Theta_{0}\left(U_{I_{0}^{\prime}} H^{\infty}(\mathbb{D}) \cap A^{+}(\mathbb{T})\right)=\mathcal{L}^{-1}\left(T_{J_{0}^{\prime}} H^{\infty}(P) \cap A_{0}(P)\right) .
$$

Thus we see that $U_{I_{0}}=U_{I_{0}^{\prime}}$ if and only if $T_{J_{0}}=T_{J_{0}^{\prime}}$. This finishes the proof since $U_{I_{0}}=U_{I}, U_{I_{0}^{\prime}}=U_{I^{\prime}}, T_{J_{0}}=T_{J}$ and $T_{J_{0}^{\prime}}=T_{J^{\prime}}$. 
Theorem 3.6. Let $s$ be a nonnegative real number, and $J$ be a closed ideal of $L^{1}\left(\mathbb{R}^{+}, \omega_{s}\right)$ such that $h_{+}^{0}(J)$ is compact and at most countable. Then

$$
\begin{aligned}
& J=\left\{f \in L^{1}\left(\mathbb{R}^{+}, \omega_{s}\right): T_{J} \mid \mathcal{L}(f) \text { and } \mathcal{L}(f)^{(k)}=0\right. \\
& \left.\qquad \text { on } h^{k}(J) \cap i \mathbb{R}, \quad(0 \leq k \leq[s])\right\} .
\end{aligned}
$$

Proof: We first suppose that $J$ is modular. For a real number $a \neq 0$ and $f \in L^{1}\left(\mathbb{R}^{+}, \omega_{s}\right)$ we set $T_{a}(f)(x)=a f(a x), x \in \mathbb{R} ; T_{a}$ is an isomorphism of $L^{1}\left(\mathbb{R}^{+}, \omega_{s}\right)$. So using this transformation we may assume without loss of generality that $h_{+}^{0}(J)$ is contained in $[0,+\infty) \times(-\pi, \pi)$. Set

$$
\begin{array}{r}
J_{0}=\left\{f \in L^{1}\left(\mathbb{R}^{+}, \omega_{s}\right): T_{J} \mid \mathcal{L}(f) \text { and } \mathcal{L}(f)^{(k)}=0\right. \\
\left.\quad \text { on } h^{k}(J), \quad(0 \leq k \leq[s])\right\} .
\end{array}
$$

We also set $I=\Theta^{-1}(J)$ and $I_{0}=\Theta^{-1}\left(J_{0}\right)$. By definition of $J_{0}$, we have $T_{J}=T_{J_{0}}$. So, we deduce from Lemma 3.5 that

$$
S_{I}=S_{I_{0}} .
$$

Furthermore, for all $0 \leq k \leq[s]$, we have $h^{k}(J)=h^{k}\left(J_{0}\right)$. So we deduce from Lemma 3.4 that

$$
h^{k}(I)=h^{k}\left(I_{0}\right), \quad(0 \leq k \leq[s]) .
$$

It follows then from (24), (25) and Theorem 3.1 that $I=I_{0}$. Since $\Theta$ is a bijection, we have $J=J_{0}$.

Suppose now that $J$ is not modular. We set $J_{1}=\delta_{-\beta_{I}} * J ; J_{1}$ is a closed ideal and we have $\beta_{J_{1}}=0, T_{J}(z)=e^{-\beta_{I} z} T_{J_{1}}(z),(z \in P)$ and $h^{k}\left(J_{1}\right)=h^{k}(J),(0 \leq k \leq[s])$. So $J_{1}$ is modular and applying the previous result to $J_{1}$, we obtain the desired equality for $J$.

\section{Further examples}

In this section, we give three further examples of algebras where, to our knowledge, the structure of closed ideals with countable hull has not been already given. For these algebras, the most difficult part is to prove that they satisfy the analytic Ditkin condition. Indeed, the fact that they satisfy conditions (H1)-(H3) is quite easy to verify and so, left to the reader. 
Recall that we denote $\alpha: z \rightarrow z$ the identity map. We will need the following sequence $\left(e_{n}\right)_{n \geq 1}$ defined by

$$
e_{n}=\frac{\alpha-1}{\alpha-1-1 / n} \quad(n \geq 1) .
$$

Notice that $\left\|e_{n}\right\|_{\infty} \leq 2$ and $\lim _{n \rightarrow \infty}\left\|e_{n} f-f\right\|_{\infty}=0$ for every function $f \in a(\mathbb{D})$ such that $f(1)=0$. Here $\|\cdot\|_{\infty}$ denotes the supremum norm on $\overline{\mathbb{D}}$.

4.1. The algebra $\boldsymbol{H}_{\boldsymbol{m}}^{\mathbf{1}}$. Let $1 \leq p<+\infty$ and $m \geq 1$ an integer. We define $H_{m}^{p}$ to be the algebra of all functions $f$ which are analytic in $\mathbb{D}$ and such that $f^{(m)} \in H^{p}$, equipped with the norm

$$
\|f\|_{p, m}=|f(0)|+\cdots+\left|f^{(m-1)}(0)\right|+\left\|f^{(m)}\right\|_{p},
$$

where $\|\cdot\|_{p}$ is the norm of $H^{p}$, the usual Hardy space in the unit disc. B. I. Korenblum showed in [17] that for $p=2$, all closed ideals of $H_{m}^{p}$ are standard. F. A. Shamoyan extended this result in $[\mathbf{2 7}]$ to all $p$ with $1<p<+\infty$. For $p=1$, N. A. Shirokov announced in [29] that a closed ideal $I$ of $H_{m}^{1}$ is standard if the following conditions are satisfied

$$
\begin{array}{ll}
\rho(z) \leq C(1-|z|)^{\gamma}, & z \in h^{0}(I) \cap \mathbb{D}, \\
\rho(z) \leq C \operatorname{dist}\left(z, h^{0}(I) \backslash\{z\}\right)^{\gamma}, & z \in h^{0}(I) \backslash h^{m-1}(I), \\
|L| \leq C \max _{z \in L} \rho(z), & \text { for any } \operatorname{arc} L \subset \mathbb{T},
\end{array}
$$

where $\rho(z)=\operatorname{dist}\left(z, h^{m-1}(I)\right), C>0$ and $\gamma>0$ are certain constants and $|L|$ is the length of $L$. Clearly these conditions are not satisfied by all closed ideals with countable hull.

Lemma 4.1. Let $m \geq 1$ be an integer. Then $H_{m}^{1}$ satisfies the strong analytic Ditkin condition.

Proof: It is easily seen that $N_{H_{m}^{1}}=m-1$. We will suppose, without loss of generality, that $z_{0}=1$. We begin to show the result for $m=1$. So, let $f \in H_{1}^{1}$ such that $f(1)=0$, we are going to prove that

$$
\lim _{n \rightarrow+\infty}\left\|\left(e_{n}-1\right) f\right\|_{1,1}=0 .
$$

We have

$$
\left[\left(e_{n}-1\right) f\right]^{\prime}=\left(e_{n}-1\right) f^{\prime}+e_{n}^{\prime} f .
$$

Since $f^{\prime} \in H^{1}(\mathbb{D})$ and $f(1)=0$, we have

$$
f\left(e^{i \theta}\right)=\int_{0}^{\theta} i e^{i t} f^{\prime}\left(e^{i t}\right) \mathrm{d} t, \quad-\pi \leq \theta \leq \pi .
$$


Furthermore, we have $e_{n}^{\prime}\left(e^{i \theta}\right)=-\frac{1}{n}\left(e^{i \theta}-1-\frac{1}{n}\right)^{-2}$. So, we have $\left[\left(e_{n}-1\right) f\right]^{\prime}\left(e^{i \theta}\right)=\left(e_{n}\left(e^{i \theta}\right)-1\right) f^{\prime}\left(e^{i \theta}\right)-\frac{1}{n}\left(e^{i \theta}-1-\frac{1}{n}\right)^{-2} \int_{0}^{\theta} i e^{i t} f^{\prime}\left(e^{i t}\right) \mathrm{d} t$.

Consequently,

(27)

$$
\begin{aligned}
&\left\|\left[\left(e_{n}-1\right) f\right]^{\prime}\right\|_{1} \leq \int_{-\pi}^{\pi}\left|\left(e_{n}\left(e^{i \theta}\right)-1\right) f^{\prime}\left(e^{i \theta}\right)\right| \mathrm{d} \theta+\frac{1}{n} \int_{-\pi}^{\pi}\left|e^{i \theta}-1-\frac{1}{n}\right|^{-2}\left(\int_{0}^{\theta}\left|f^{\prime}\left(e^{i t}\right)\right| \mathrm{d} t\right) \mathrm{d} \theta \\
& \leq \int_{-\pi}^{\pi}\left|\left(e_{n}\left(e^{i \theta}\right)-1\right) f^{\prime}\left(e^{i \theta}\right)\right| \mathrm{d} \theta \\
& \quad+\int_{-\pi}^{\pi}\left|f^{\prime}\left(e^{i t}\right)\right|\left(\frac{1}{n} \int_{|t|}^{\pi}\left|e^{i \theta}-1-\frac{1}{n}\right|^{-2} \mathrm{~d} \theta\right) \mathrm{d} t .
\end{aligned}
$$

Now, an easy calculation and Cauchy formulae show that

$$
\begin{aligned}
\frac{1}{n} \int_{|t|}^{\pi}\left|e^{i \theta}-1-\frac{1}{n}\right|^{-2} \mathrm{~d} \theta & \leq \frac{1}{n} \int_{0}^{\pi}\left|e^{i \theta}-1-\frac{1}{n}\right|^{-2} \mathrm{~d} \theta \\
& =\frac{1}{2 i n} \int_{|\zeta|=1} \frac{1}{\left(\zeta-1-\frac{1}{n}\right)\left(1-\left(1-\frac{1}{n}\right) \zeta\right)} \mathrm{d} \zeta \\
& =\frac{n}{2 n+1} \frac{\pi}{2} \leq \frac{\pi}{2} .
\end{aligned}
$$

So, using the dominated convergence theorem of Lebesgue, we deduce from the above inequality, the fact that $f^{\prime} \in H^{1}(\mathbb{D})$ and $(27)$ that

$$
\lim _{n \rightarrow+\infty}\left\|\left[\left(e_{n}-1\right) f\right]^{\prime}\right\|_{1}=0 .
$$

Moreover it is easily seen that

$$
\lim _{n \rightarrow+\infty}\left|\left[\left(e_{n}-1\right) f\right](0)\right|=0 .
$$

So we deduce from (28) and (29) that (26) is satisfied. Now, suppose that $m \geq 2$. For all $n \geq 1$, set $\tau_{n}=e_{n}^{m}$ and let $f \in H_{m}^{1}$ such that 
$f(1)=\cdots=f^{(m-1)}(1)=0$. We have

$$
\begin{aligned}
\left\|\left(\tau_{n}-1\right) f\right\|_{1, m}=\left|\left[\left(\tau_{n}-1\right) f\right](0)\right|+\cdots & +\left|\left[\left(\tau_{n}-1\right) f\right]^{(m-2)}(0)\right| \\
& +\left\|\left[\left(\tau_{n}-1\right) f\right]^{(m-1)}\right\|_{1,1} .
\end{aligned}
$$

Since for all $0 \leq j \leq m-2$, we have clearly

$$
\lim _{n \rightarrow+\infty}\left|\left[\left(\tau_{n}-1\right) f\right]^{(j)}(0)\right|=0,
$$

it remains to prove that

$$
\lim _{n \rightarrow+\infty}\left\|\left[\left(\tau_{n}-1\right) f\right]^{(m-1)}\right\|_{1,1}=0 .
$$

One can show by induction on $k \geq 1$ that

$$
\left(\tau_{n}-1\right)^{(k)}=\left(e_{n}-1\right)(\alpha-1)^{-k} P_{k}\left(e_{n}\right) \quad(k \geq 0),
$$

where $P_{k}, k \geq 0$, are polynomials independent of $n \geq 1$. We have

$$
\begin{aligned}
& {\left[\left(\tau_{n}-1\right) f\right]^{(m-1)}=\left(\tau_{n}-1\right) f^{(m-1)}} \\
& \quad+\sum_{k=1}^{m-1}\left(\begin{array}{c}
m-1 \\
k
\end{array}\right)\left(\tau_{n}-1\right)^{(k)} f^{(m-1-k)} .
\end{aligned}
$$

We deduce from (26) that for all $f \in\left\{g \in H_{1}^{1}: g(1)=0\right\}$, there exists $C>0$ such that $\left\|e_{n} f\right\|_{1,1} \leq C\|f\|_{1,1}$. So we deduce from the BanachSteinhaus Theorem that there exists $C^{\prime}>0$ such that

$$
\left\|e_{n} f\right\|_{1,1} \leq C^{\prime}\|f\|_{1,1} \quad\left(n \geq 1, f \in\left\{g \in H_{1}^{1}: g(1)=0\right\}\right) .
$$

For all $0 \leq j \leq m-1, f^{(j)} \in\left\{g \in H_{1}^{1}: g(1)=0\right\}$. And since $P_{k}$, $k \geq 0$, are polynomials independent of $n \geq 1$, we deduce from (30), (31) and (32) that there exists $K>0$ such that

$$
\begin{aligned}
& \left\|\left[\left(\tau_{n}-1\right) f\right]^{(m-1)}\right\|_{1,1} \leq K\left\|\left(e_{n}-1\right) f^{(m-1)}\right\|_{1,1} \\
& \quad+K \sum_{k=1}^{m-1}\left\|\left(e_{n}-1\right) f^{(m-1-k)}\right\|_{1,1} .
\end{aligned}
$$

So we deduce from $(26)$ that $\lim _{n \rightarrow+\infty}\left\|\left[\left(\tau_{n}-1\right) f\right]^{(m-1)}\right\|_{1,1}=0$, which concludes the proof. 
4.2. The algebra $\mathcal{F} \ell^{p}(\mathbb{N}, s)$. Let $1 \leq p<+\infty$ and $s>0$ be real numbers. We denote by $\mathcal{F} \ell^{p}(\mathbb{N}, s)$ the space of all continuous functions on $\mathbb{T}$ such that

$$
\|f\|_{p, s}=\left(\sum_{n=0}^{+\infty}|\hat{f}(n)|^{p}(1+n)^{s p}\right)^{\frac{1}{p}}<+\infty .
$$

Note that $\mathcal{F} \ell^{1}(\mathbb{N}, s)=A_{s}^{+}(\mathbb{T})$ (see previous section). Let $1<p^{\prime} \leq+\infty$ such that $\frac{1}{p}+\frac{1}{p^{\prime}}=1$. It is well known that $\left(\mathcal{F} \ell^{p}(\mathbb{N}, s),\|\|_{p, s}\right)$ is a Banach algebra if and only if $s-\frac{1}{p^{\prime}}>0$ (see [5]). A standard computation shows that

$$
\begin{aligned}
N_{\mathcal{F} \ell^{p}(\mathbb{N}, s)} & = \begin{cases}{\left[s-\frac{1}{p^{\prime}}\right]} & \text { if } s-\frac{1}{p^{\prime}} \notin \mathbb{N} \\
s-\frac{1}{p^{\prime}}-1 & \text { if } s-\frac{1}{p^{\prime}} \in \mathbb{N}\end{cases} \\
& =-\left[-\left(s-\frac{1}{p^{\prime}}\right)+1\right] .
\end{aligned}
$$

Lemma 4.2. Assume that $0<s-\frac{1}{p^{\prime}}<1$. Then $\mathcal{F} \ell^{p}(\mathbb{N}, s)$ satisfies the strong analytic Ditkin condition.

Proof: Let $f \in \mathcal{F} \ell^{p}(\mathbb{N}, s)$ such that $f(1)=0$. For $n \geq 1$, we have

$$
e_{n}=1-\frac{1}{n+1} \sum_{k=0}^{+\infty}\left(\frac{n}{n+1}\right)^{k} \alpha^{k}
$$

and

with

$$
\left(e_{n}-1\right) f=\sum_{k=0}^{+\infty} a_{k} \alpha^{k}
$$

$$
a_{k}=-\frac{1}{n+1} \sum_{j=0}^{k}\left(\frac{n}{n+1}\right)^{k-j} \hat{f}(j), \quad k \geq 0 .
$$

Since $f(1)=\sum_{j=0}^{+\infty} \hat{f}(j)=0$, we get for all $k \geq 0$,

$$
\begin{aligned}
\left|a_{k}\right|^{p} & =\frac{1}{(n+1)^{p}}\left|\sum_{j=0}^{k}\left(\frac{n}{n+1}\right)^{k-j} \hat{f}(j)\right|^{p} \\
& =\frac{1}{(n+1)^{p}}\left(\frac{n}{n+1}\right)^{k p}\left|\sum_{j=0}^{k}\left[\left(\frac{n+1}{n}\right)^{j}-1\right] \hat{f}(j)-\sum_{j=k+1}^{+\infty} \hat{f}(j)\right|^{p} .
\end{aligned}
$$


Hence

$$
\left\|\left(e_{n}-1\right) f\right\|^{p}=\frac{1}{(n+1)^{p}} \sum_{k=0}^{+\infty}\left(\frac{n}{n+1}\right)^{k p} A_{k}(1+k)^{s p},
$$

where

$$
A_{k}=\left|\sum_{j=0}^{k}\left[\left(\frac{n+1}{n}\right)^{j}-1\right] \hat{f}(j)-\sum_{j=k+1}^{+\infty} \hat{f}(j)\right|^{p} .
$$

By the Hölder inequality, we obtain

$$
\begin{aligned}
A_{k} \leq\left(u_{k}+v_{k}\right)\left[\sum_{j=0}^{k}\left[\left(\frac{n+1}{n}\right)^{j}-1\right]^{s p-p / p^{\prime}} \min \left(1, \frac{j}{n}\right)^{p / p^{\prime}}|\hat{f}(j)|^{p}\right. \\
\left.+\frac{1}{(n+1)^{p / p^{\prime}}(k+1)^{s p-p / p^{\prime}}} \sum_{j=k+1}^{+\infty}(1+j)^{s p}|\hat{f}(j)|^{p}\right]
\end{aligned}
$$

where

$$
\begin{aligned}
u_{k} & =\sum_{j=0}^{k}\left[\left(\frac{n+1}{n}\right)^{j}-1\right]^{p^{\prime}+1-s p^{\prime}} \min \left(1, \frac{j}{n}\right)^{-1} \\
\text { and } \quad v_{k} & =(n+1)(k+1)^{s p^{\prime}-1} \sum_{j=k+1}^{+\infty}(1+j)^{-s p^{\prime}}
\end{aligned}
$$

We have clearly $v_{k} \leq \frac{1}{s p^{\prime}-1}(n+1)$. We write

$u_{k}=\sum_{j=0}^{n-1}\left[\left(\frac{n+1}{n}\right)^{j}-1\right]^{p^{\prime}+1-s p^{\prime}}\left(\frac{j}{n}\right)^{-1}+\sum_{j=n}^{k}\left[\left(\frac{n+1}{n}\right)^{j}-1\right]^{p^{\prime}+1-s p^{\prime}}$

with the understanding that " $\sum_{j=n}^{k} "=0$ if $k<n$. Since $\left(\frac{n+1}{n}\right)^{j}-1 \leq$ $\min \left(1, \frac{j}{n}\right)\left(\frac{n+1}{n}\right)^{j}$ and $\left(\frac{n+1}{n}\right)^{j} \leq e$ if $0 \leq j \leq n-1$, we get $u_{k} \leq e^{p^{\prime}+1-s p^{\prime}} \sum_{j=0}^{n-1}\left(\frac{j}{n}\right)^{p^{\prime}-s p^{\prime}}+\sum_{j=n}^{k}\left(\frac{n+1}{n}\right)^{j\left(p^{\prime}+1-s p^{\prime}\right)}$

$$
\leq e^{p^{\prime}+1-s p^{\prime}} \sum_{j=0}^{n-1}\left(\frac{j}{n}\right)^{p^{\prime}-s p^{\prime}}+e^{p^{\prime}+1-s p^{\prime}} \sum_{j=n}^{k}\left(\frac{n+1}{n}\right)^{(j-n)\left(p^{\prime}+1-s p^{\prime}\right)} .
$$


Since $p^{\prime}-s p^{\prime}>-1$, it follows from the last inequality that

$$
\begin{aligned}
u_{k} & \leq e^{p^{\prime}+1-s p^{\prime}}(n+1)+e^{p^{\prime}+1-s p^{\prime}}(n+1)\left[\left(\frac{n+1}{n}\right)^{(k-n+1)\left(p^{\prime}+1-s p^{\prime}\right)}-1\right] \\
& \lesssim e^{p^{\prime}+1-s p^{\prime}}(n+1)\left(\frac{n+1}{n}\right)^{k\left(p^{\prime}+1-s p^{\prime}\right)},
\end{aligned}
$$

where the notation $A \lesssim B$ means that there exists a constant $C>0$, independent of $n, k$ and $f$ such that $A \leq C B$.

So

$$
\begin{aligned}
A_{k} \lesssim(n+1)^{p / p^{\prime}}\left(\frac{n+1}{n}\right)^{k\left(p+p / p^{\prime}-s p\right)} \\
\times\left[\sum_{j=0}^{k}\left[\left(\frac{n+1}{n}\right)^{j}-1\right]^{s p-p / p^{\prime}} \min \left(1, \frac{j}{n}\right)^{p / p^{\prime}}|\hat{f}(j)|^{p}\right. \\
\left.\quad+\frac{1}{(n+1)^{p / p^{\prime}}(k+1)^{s p-p / p^{\prime}}} \sum_{j=k+1}^{+\infty}(1+j)^{s p}|\hat{f}(j)|^{p}\right] .
\end{aligned}
$$

Thus we get

$$
\begin{aligned}
\left\|\left(e_{n}-1\right) f\right\|^{p} \lesssim & (n+1)^{p / p^{\prime}-p} \\
& \times \sum_{j=0}^{+\infty}\left[\left(\frac{n+1}{n}\right)^{j}-1\right]^{s p-p / p^{\prime}} \min \left(1, \frac{j}{n}\right)^{p / p^{\prime}}|\hat{f}(j)|^{p} \\
& \times\left(\sum_{k=j}^{+\infty}\left(\frac{n}{n+1}\right)^{k\left(s p-p / p^{\prime}\right)}(1+k)^{s p}\right) \\
+ & +1)^{-p} \sum_{j=0}^{+\infty}(1+j)^{s p}|\hat{f}(j)|^{p} \\
& \times\left(\sum_{k=0}^{j-1}\left(\frac{n}{n+1}\right)^{k\left(s p-p / p^{\prime}\right)}(1+k)^{p / p^{\prime}}\right) .
\end{aligned}
$$


Let $\beta \geq 0$ be a real number and $j \geq 0$ an integer. Then there exists a constant $C>0$ such that for every real number $x$ with $0 \leq x<1$,

$$
\sum_{k=j}^{+\infty}(1+k)^{\beta} x^{k} \leq C \frac{x^{j}}{1-x} \max \left(j+1, \frac{1}{1-x}\right)^{\beta} .
$$

Applying this inequality for $\beta=s p$ and $x=\left(\frac{n}{n+1}\right)^{s p-p / p^{\prime}}$ and using the fact that $\left(\frac{n+1}{n}\right)^{j}-1 \leq\left(\frac{n+1}{n}\right)^{j} \min \left(1, \frac{j}{n}\right)$, we obtain

(35) $\left\|\left(e_{n}-1\right) f\right\|^{p} \lesssim \sum_{j=0}^{+\infty} \min \left(1, \frac{j}{n}\right)^{s p}|\hat{f}(j)|^{p} \max (j+1, n+1)^{s p}$

$$
+(n+1)^{-p} \sum_{j=0}^{+\infty}(1+j)^{s p}|\hat{f}(j)|^{p}\left(\sum_{k=0}^{j-1}\left(\frac{n}{n+1}\right)^{k\left(s p-p / p^{\prime}\right)}(1+k)^{p / p^{\prime}}\right) .
$$

Using again (34), we obtain

$$
\begin{aligned}
\sum_{k=0}^{j-1}\left(\frac{n}{n+1}\right)^{k\left(s p-p / p^{\prime}\right)}(1+k)^{p / p^{\prime}} & \leq \sum_{k=0}^{+\infty}\left(\frac{n}{n+1}\right)^{k\left(s p-p / p^{\prime}\right)}(1+k)^{p / p^{\prime}} \\
& \lesssim(n+1)^{p / p^{\prime}+1}=(n+1)^{p} .
\end{aligned}
$$

It follows now from (35) and the Lebesgue dominated convergence theorem that

$$
\lim _{n \rightarrow \infty}\left\|\left(e_{n}-1\right) f\right\|=0 .
$$

Remark 4.3. For $s=1+1 / p^{\prime}$, we have $N_{\mathcal{F} \ell^{p}(\mathbb{N}, s)}=0$. But in this case we have

$$
\left\|\left(e_{n}-1\right)(\alpha-1)\right\|^{p}=\frac{1}{(n+1)^{p}}+\frac{1}{n^{p}(n+1)^{p}} \sum_{k=1}^{+\infty}\left(\frac{n}{n+1}\right)^{k p}(1+k)^{\alpha p}
$$

which implies that

$$
\left\|\left(e_{n}-1\right)(\alpha-1)\right\| \nrightarrow 0, \text { as } n \rightarrow 0 .
$$

We get this with the help of the following inequality:

$$
\frac{1}{(1-x)^{\beta+1}} \leq 1+(1+\beta) \sum_{k \geq 1} k^{\beta} x^{k}, \quad(\beta \geq 0,0 \leq x<1) .
$$


4.3. The algebra $\boldsymbol{\lambda}_{\boldsymbol{\omega}}$. Let $w$ be a nonnegative nondecreasing subadditive function on $(0,+\infty)$ such that $w(0+)=0$. Let $p$ be a nonnegative integer. We define the Banach algebra

$\lambda_{w}^{(p)}=\left\{f \in a^{p}(\mathbb{D}):\left|f^{(p)}(z)-f^{(p)}\left(z^{\prime}\right)\right|=o\left(w\left(\left|z-z^{\prime}\right|\right)\right),\left|z-z^{\prime}\right| \rightarrow 0\right\}$,

equipped with the norm $\|f\|_{\lambda_{w, p}}=\|f\|_{\mathcal{C}^{p}(\mathbb{T})}+\sup _{z, z^{\prime} \in \mathbb{T}} \frac{\left|f^{(p)}(z)-f^{(p)}\left(z^{\prime}\right)\right|}{w\left(\left|z-z^{\prime}\right|\right)}$.

We set $\lambda_{w}^{(0)}=\lambda_{w}$. When $p \geq 1$, all closed ideals of $\lambda_{w}^{(p)}$ are standard, but when $p=0$, this holds if $w(x)=O\left(x^{\alpha}\right), x \rightarrow 0$, for some $\alpha \in(0,1]$ or if $w$ satisfies the Zygmund condition, that is

$$
\int_{0}^{\delta} \frac{w(x)}{x} \mathrm{~d} x=o(w(\delta)), \quad \delta \rightarrow 0
$$

(see $[\mathbf{2 8}],[\mathbf{3 0}],[\mathbf{1 9}])$. For $\alpha>0$, we set

$$
w_{\alpha}(x)=\frac{1}{\left(\left|\log \frac{1}{x}\right|+1\right)^{\alpha}}, \quad x>0 .
$$

There is no characterisation of closed ideals of the algebras $\lambda_{w_{\alpha}}$ (see the introduction of [30]). For this reason we consider here this algebra.

Let us introduce the following condition:

$$
\text { There exists } C>0 \text {, such that } \frac{w(y)}{w(x)} \leq C \frac{y}{x}, \quad 0<x \leq y .
$$

This condition is satisfied by concave functions and in particular by functions $w_{\alpha}, \alpha>0$.

Lemma 4.4. Assume that $w$ satisfies condition (36). Then $\lambda_{w}$ satisfies the strong analytic Ditkin condition.

Proof: We have $N_{\lambda_{w}}=0$. Let $f \in \lambda_{w}$ such that $f(1)=0$, we have to prove that

$$
\lim _{n \rightarrow+\infty} \sup _{z, z^{\prime} \in \mathbb{T}} \frac{\left|\left[\left(e_{n}-1\right) f\right](z)-\left[\left(e_{n}-1\right) f\right]\left(z^{\prime}\right)\right|}{w\left(\left|z-z^{\prime}\right|\right)}=0 .
$$

For $z, z^{\prime} \in \mathbb{T}$ and $n \geq 1$, set

$$
A_{n}\left(z, z^{\prime}\right)=\frac{\left[\left(e_{n}-1\right) f\right](z)-\left[\left(e_{n}-1\right) f\right]\left(z^{\prime}\right)}{w\left(\left|z-z^{\prime}\right|\right)} .
$$

Let $\varepsilon>0$. Since $f \in \lambda_{w}$, there exists $\eta>0$ such that

$$
\left|z-z^{\prime}\right| \leq \eta \Longrightarrow \frac{\left|f(z)-f\left(z^{\prime}\right)\right|}{w\left(\left|z-z^{\prime}\right|\right)} \leq \varepsilon .
$$


Let $z, z^{\prime} \in \mathbb{T}$. We will distinguish two cases:

Case 1: $|z-1|>\frac{\eta}{2}$ and $\left|z^{\prime}-1\right|>\frac{\eta}{2}$.

An easy calculation shows that $A_{n}\left(z, z^{\prime}\right)=B_{n}\left(z, z^{\prime}\right)+C_{n}\left(z, z^{\prime}\right)$, where

$$
\begin{aligned}
B_{n}\left(z, z^{\prime}\right) & =\left(e_{n}(z)-1\right) \frac{f(z)-f\left(z^{\prime}\right)}{w\left(\left|z-z^{\prime}\right|\right)} \\
\text { and } \quad C_{n}\left(z, z^{\prime}\right) & =\frac{f\left(z^{\prime}\right)}{w\left(\left|z-z^{\prime}\right|\right)}\left(e_{n}(z)-e_{n}\left(z^{\prime}\right)\right) .
\end{aligned}
$$

Since $\lim _{n \rightarrow+\infty} \sup _{|\xi-1|>\frac{\eta}{2}}\left|e_{n}(\xi)-1\right|=0$, it is easily seen that there exists $n_{1} \geq 0$ such that, for all $n \geq n_{1},\left|B_{n}\left(z, z^{\prime}\right)\right| \leq \varepsilon$. On the other hand side we have

$$
e_{n}(z)-e_{n}\left(z^{\prime}\right)=\frac{z-z^{\prime}}{n(z-1-1 / n)\left(z^{\prime}-1-1 / n\right)},
$$

which implies that $\left|e_{n}(z)-e_{n}\left(z^{\prime}\right)\right| \leq \frac{4\left|z-z^{\prime}\right|}{n \eta^{2}}$. Moreover condition (36), implies that $\frac{\left|z-z^{\prime}\right|}{w\left(\left|z-z^{\prime}\right|\right)} \leq C^{\prime}$, where $C^{\prime}=\frac{2}{C w(2)}$. So there exists $n_{2} \geq 0$ such that, for all $n \geq n_{2},\left|C_{n}\left(z, z^{\prime}\right)\right| \leq \varepsilon$. So, in this case, there exists $n_{3}=\max \left\{n_{1}, n_{2}\right\}$ such that, for all $n \geq n_{3},\left|A_{n}\left(z, z^{\prime}\right)\right| \leq 2 \varepsilon$.

Case $2:|z-1| \leq \frac{\eta}{2}$ or $\left|z^{\prime}-1\right| \leq \frac{\eta}{2}$.

Without loss of generality, we can suppose that $\left|z^{\prime}-1\right| \leq \frac{\eta}{2}$. If $\left|z-z^{\prime}\right| \leq \eta$, we deduce from (38) that $\left|B_{n}\left(z, z^{\prime}\right)\right| \leq 3 \varepsilon$. If $\left|z-z^{\prime}\right|>\eta$, then $|z-1|>\frac{\eta}{2}$, and we can find, as in the previous case, $n_{4} \geq 0$ such that, for all $n \geq n_{4},\left|B_{n}\left(z, z^{\prime}\right)\right| \leq \varepsilon$. Now, we have to estimate $C_{n}\left(z, z^{\prime}\right)$. Since $\left|z^{\prime}-1\right| \leq \frac{\eta}{2}$ and $f(1)=0$, we have by $(38),\left|f\left(z^{\prime}\right)\right| \leq \varepsilon \omega\left(\left|z^{\prime}-1\right|\right)$, and so

$$
\left|C_{n}\left(z, z^{\prime}\right)\right| \leq \varepsilon \frac{\omega\left(\left|z^{\prime}-1\right|\right)}{\omega\left(\left|z-z^{\prime}\right|\right)} \frac{\left|z-z^{\prime}\right|}{n|z-1-1 / n|\left|z^{\prime}-1-1 / n\right|}
$$


Suppose that $\left|z^{\prime}-1\right| \geq\left|z-z^{\prime}\right|$. By condition (36), we have $\frac{w\left(\left|z^{\prime}-1\right|\right)}{w\left(\left|z-z^{\prime}\right|\right)} \leq$ $C \frac{\left|z^{\prime}-1\right|}{\left|z-z^{\prime}\right|}$, so that

$$
\begin{aligned}
\left|C_{n}\left(z, z^{\prime}\right)\right| & \leq C \varepsilon \frac{\left|z^{\prime}-1\right|}{n|z-1-1 / n|\left|z^{\prime}-1-1 / n\right|} \\
& \leq C \varepsilon .
\end{aligned}
$$

Suppose that $\left|z^{\prime}-1\right|<\left|z-z^{\prime}\right|$. Then, since $w$ is nondecreasing, $\frac{w\left(\left|z^{\prime}-1\right|\right)}{w\left(\left|z-z^{\prime}\right|\right)} \leq 1$. Furthermore, a standard computation shows that

$$
\begin{aligned}
\frac{\left|z-z^{\prime}\right|}{n|z-1-1 / n|\left|z^{\prime}-1-1 / n\right|} \leq\left|e_{n}(z)\right|+\left|e_{n}\left(z^{\prime}\right)\right| & \leq 2, \\
& \left(n \geq 1 \text { and } z, z^{\prime} \in \mathbb{T}\right) .
\end{aligned}
$$

So we deduce from (41) that $\left|C_{n}\left(z, z^{\prime}\right)\right| \leq 2 \varepsilon$. In this case, for all $n \geq n_{4}$, we have $\left|A_{n}\left(z, z^{\prime}\right)\right| \leq C_{1} \varepsilon$, where $C_{1}=3+\max \{2, C\}$.

Finally, if we set $n_{0}=\max \left\{n_{3}, n_{4}\right\}$, we have

$$
\left|A_{n}\left(z, z^{\prime}\right)\right| \leq C_{1} \varepsilon, \quad\left(n \geq n_{0} \text { and } z, z^{\prime} \in \mathbb{T}\right),
$$

which proves (37).

Theorem 4.5. Let $\mathcal{B}$ be one of the following algebras:

(a) $H_{m}^{1}$, for $m \geq 1$ an integer.

(b) $\mathcal{F} \ell^{p}(\mathbb{N}, s)$, for $1<p<+\infty$ and $0<s-\frac{1}{p^{\prime}}<1$.

(c) $\lambda_{\omega}$, for $\omega$ satisfying condition (36).

Let $I$ be a closed ideal of $\mathcal{B}$ such that $h^{0}(I)$ is at most countable. Then $I$ is standard.

Proof: It is easily checked that $\mathcal{B}$ is continuously imbedded in $a(\mathbb{D})$ and satisfies conditions (H1)-(H3). Now the theorem follows immediately from Lemmas 4.1, 4.2, 4.4, and Theorem 2.11.

\section{References}

[1] C. Agrafeuil, Idéaux fermés de certaines algèbres de Beurling et application aux opérateurs à spectre dénombrable, Studia Math. 167(2) (2005), 133-151.

[2] A. Atzmon, Operators which are annihilated by analytic functions and invariant subspaces, Acta Math. 144(1-2) (1980), 27-63. 
[3] C. Bennett and J. E. Gilbert, Homogeneous algebras on the circle. I. Ideals of analytic functions, Ann. Inst. Fourier (Grenoble) 22(3) (1972), 1-19.

[4] O. El-Fallah, Idéaux fermés de $L^{1}\left(\mathbb{R}_{+}\right)$, Math. Scand. 72(1) (1993), 120-130.

[5] O. El-Fallah, N. K. Nikol'skiI, and M. Zarrabi, Estimates for resolvents in Beurling-Sobolev algebras, (Russian), Algebra $i$ Analiz 10(6) (1998), 1-92; translation in: St. Petersburg Math. J. 10(6) (1999), 901-964.

[6] J. Esterle, Distributions on Kronecker sets, strong forms of uniqueness, and closed ideals of $A^{+}$, J. reine angew. Math. 450 (1994), 43-82.

[7] J. Esterle, E. Strouse, and F. Zounkia, Closed ideals of $A^{+}$ and the Cantor set, J. reine angew. Math. 449 (1994), 65-79.

[8] V. M. FAĬVYŠEvsKIĬ, The structure of the ideals of certain algebras of analytic functions, (Russian), Dokl. Akad. Nauk SSSR 211 (1973), 537-539; translation in: Soviet Math. Dokl. 14 (1973), 1067-1070.

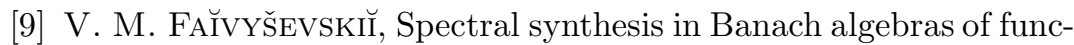
tions analytic in the disc, (Russian), Funkcional. Anal. i Priložen 8(3) (1974), 85-86; translation in: Functional Anal. Appl. 8 (1974), 268-269.

[10] T. W. Gamelin, "Uniform algebras", Prentice-Hall, Inc., Englewood Cliffs, N. J., 1969.

[11] V. P. GurARII , Spectral synthesis of bounded functions on the half axis, (Russian), Funkcional. Anal. i Priložen 3(4) (1969), 34-48.

[12] V. P. Gurarĭ, Harmonic analysis in spaces with weight, (Russian), Trudy Moskov. Mat. Obšč 35 (1976), 21-76; translation in: Trans. Moscow Math. Soc. 35 (1979), 21-75.

[13] H. Hedenmalm, A comparison between the closed modular ideals in $l^{1}(w)$ and $L^{1}(w)$, Math. Scand. 58(2) (1986), 275-300.

[14] K. Hoffman, "Banach spaces of analytic functions", Prentice-Hall Series in Modern Analysis, Prentice-Hall, Inc., Englewood Cliffs, N. J., 1962.

[15] J.-P. Kahane, Idéaux primaires fermés dans certaines algèbres de Banach de fonctions analytiques, in: " $L$ 'analyse harmonique dans le domaine complexe" (Actes Table Ronde Internat., CNRS, Montpellier, 1972), Lecture Notes in Math. 336, Springer, Berlin, 1973, pp. $5-14$.

[16] Y. Katznelson, "An introduction to harmonic analysis", John Wiley \& Sons, Inc., New York-London-Sydney, 1968. 
[17] B. I. Korenblum, Invariant subspaces of the shift operator in a weighted Hilbert space, (Russian), Mat. Sb. (N.S.) 89(131) (1972), 110-137, 166; translation in: Math. USSR-Sb. 18 (1972), 111-138.

[18] B. I. Korenblum, Closed ideals of the ring $A^{n}$, (Russian), Funkcional. Anal. i Priložen 6(3) (1972), 38-52; translation in: Functional Anal. Appl. 6 (1972), 203-214.

[19] A. L. Matheson, Closed ideals in rings of analytic functions satisfying a Lipschitz condition, in: "Banach spaces of analytic functions" (Proc. Pelczynski Conf., Kent State Univ., Kent, Ohio, 1976), Lecture Notes in Math. 604, Springer, Berlin, 1977, pp. $67-72$.

[20] H. Mirkil, "The work of Šilov on commutative semi-simple Banach algebras", Second revised edition, Notas de Matemática 20, Instituto de Matemática Pura e Aplicada do Conselho Nacional de Pesquisas, Rio de Janeiro, 1966.

[21] N. K. Nikol'skII, "Treatise on the shift operator", Spectral function theory, With an appendix by S. V. Hruščev and V. V. Peller, Translated from the Russian by Jaak Peetre, Grundlehren der Mathematischen Wissenschaften 273, Springer-Verlag, Berlin, 1986.

[22] B. Nyman, On the One-Dimensional Translation Group and SemiGroup in Certain Function Spaces, Thesis, University of Uppsala (1950).

[23] T. V. Pedersen, Some results about Beurling algebras with applications to operator theory, Studia Math. 115(1) (1995), 39-52.

[24] W. Rudin, The closed ideals in an algebra of analytic functions, Canad. J. Math. 9 (1957), 426-434.

[25] F. A. Shamoyan, Construction of a certain special sequence, and structure of the closed ideals in certain algebras of analytic functions, (Russian), Izv. Akad. Nauk Armjan SSR Ser. Mat. 7(6) (1972), 440-470, 479.

[26] F. A. Shamoyan, The structure of closed ideals in certain algebras of functions analytic in the disc and smooth up to its boundary, (Russian), Akad. Nauk Armjan. SSR Dokl. 60(3) (1975), 133-136.

[27] F. A. Shamoyan, Closed ideals in algebras of analytic functions that are smooth up to the boundary, (Russian), Izv. Akad. Nauk Armyan. SSR Ser. Mat. 16(3) (1981), 173-191, 247; translation in: Soviet J. Contemporary Math. Anal. 16(2) (1981), 173-191.

[28] F. A. Shamoyan, Closed ideals in algebras of functions that are analytic in the disk and smooth up to its boundary, (Russian), Mat. 
Sb. 184(8) (1993), 109-136; translation in: Russian Acad. Sci. Sb. Math. 79(2) (1994), 425-445.

[29] N. A. Shirokov, Standard ideals of the algebra $H_{n}^{1}$, (Russian), Funktsional. Anal. i Prilozhen. 13(1) (1979), 86-87; translation in: Functional Anal. Appl. 13(1) (1979), 73-74.

[30] N. A. Shirokov, Closed ideals of algebras of type $B_{p q}^{\alpha}$, (Russian), Izv. Akad. Nauk SSSR Ser. Mat. 46(6) (1982), 1316-1332, 1344; translation in: Math. USSR-Izv. 21 (1983), 585-600.

[31] G. E. Šilov, Homogeneous rings of functions, Amer. Math. Soc. Transl. 92 (1953), 65 pp.

[32] B. A. TAYlor AND D. L. Williams, Ideals in rings of analytic functions with smooth boundary values, Canad. J. Math. 22 (1970), $1266-1283$.

Cyril Agrafeuil:

59, rue du Moulin-Vert

75014 Paris

France

E-mail address: cyril.agrafeuil@gmail.com

Mohamed Zarrabi:

Université Bordeaux 1

Laboratoire Bordelais d'Analyse et de Géométrie (UMR 5467)

351, cours de la Libération

33405 Talence Cedex

France

E-mail address: Mohamed.Zarrabi@math.u-bordeaux1.fr

Primera versió rebuda el 13 de febrer de 2006 , darrera versió rebuda el 19 de setembre de 2007. 